\begin{abstract}
كارزدَى به عنوان سندرم روانشناختى عامل بيش بينى كننده

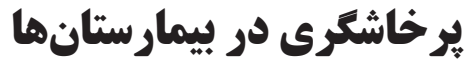

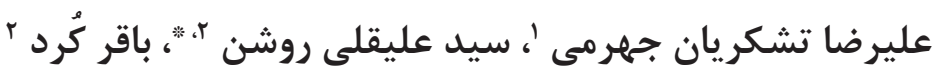

' دانشـجوى دكتـــاى مديريست، دانشـكده مديريـت و اقتصـاد، دانشـحًاه سيسـتان و بلوجسـتان،

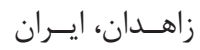

' عضو هيئت علمى دانشكده مديريت و اقتصاد، دانشخاه سيستان و بلوحستان، زاهدان، ايران
\end{abstract}

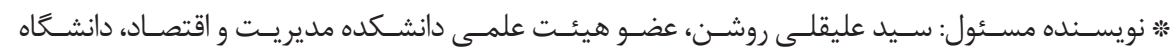

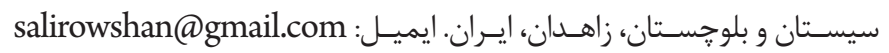

DOI: $10.21859 / \mathrm{nmj}-25023$

קقكيده

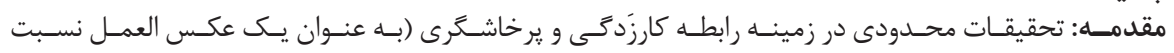

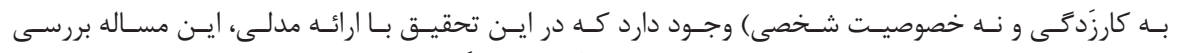

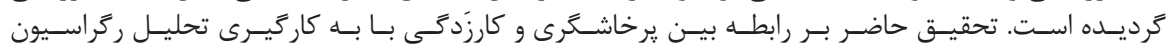

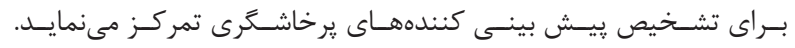

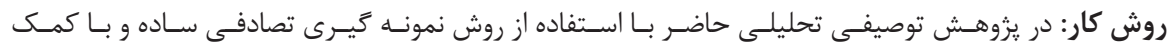

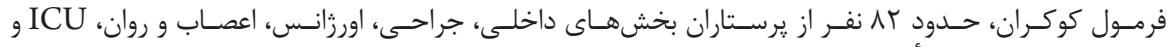
ير CCU

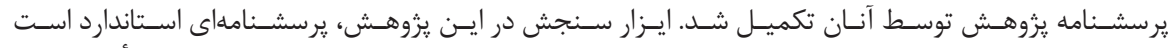

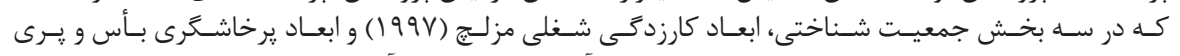

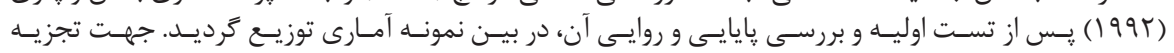

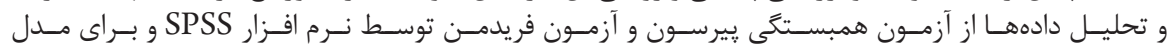

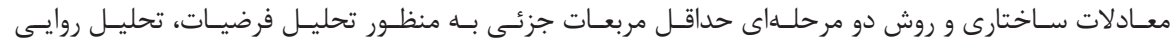

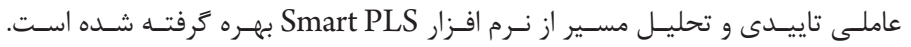

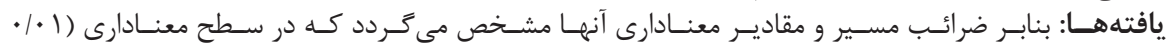

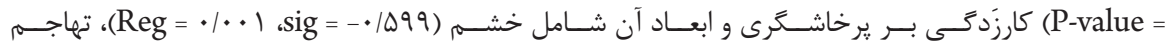

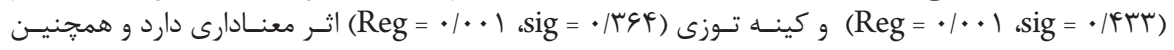

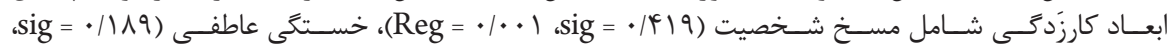

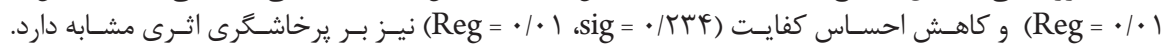

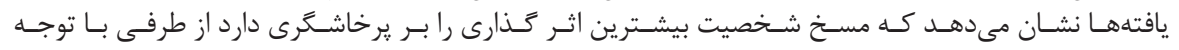

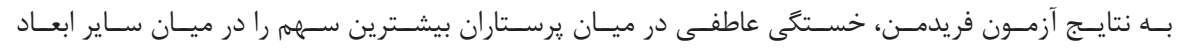

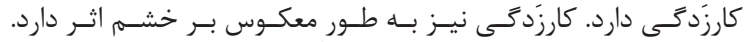

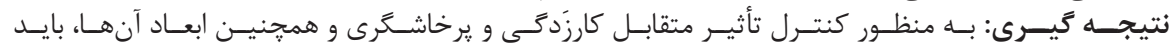

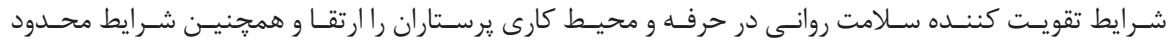

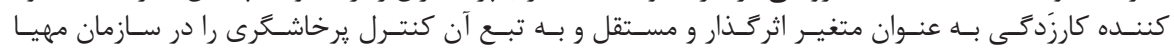

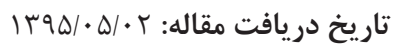

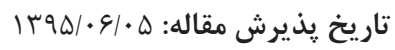

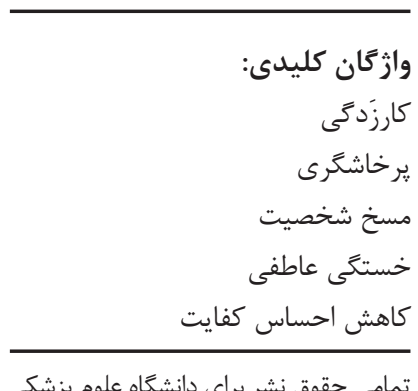
تمدامى حقوق نشر براى دانشكاه علوم يزشكى

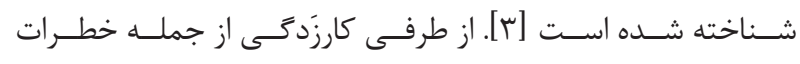

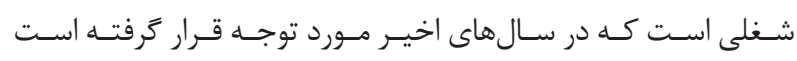

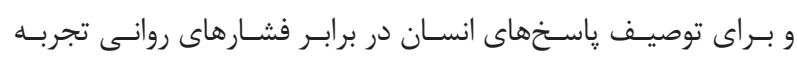

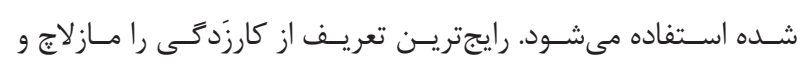

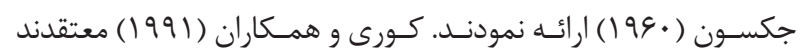

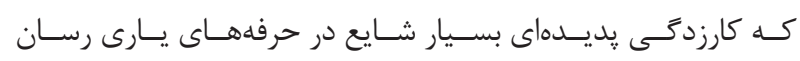

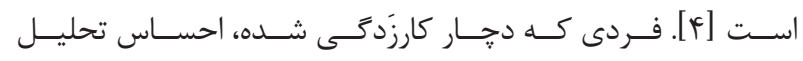

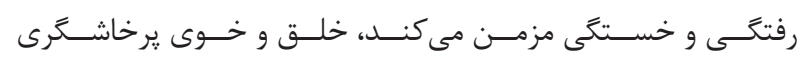

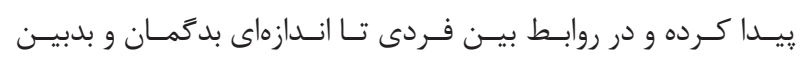

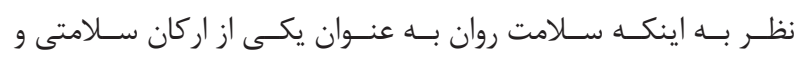

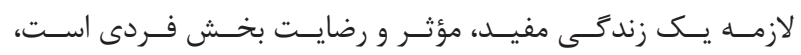

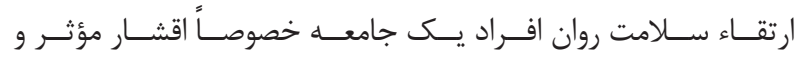

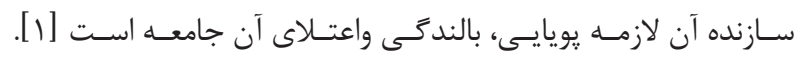

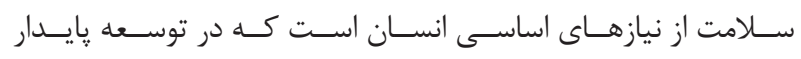

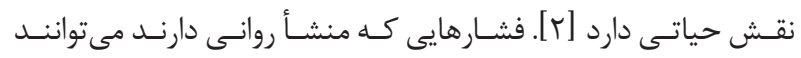

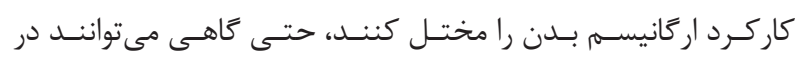

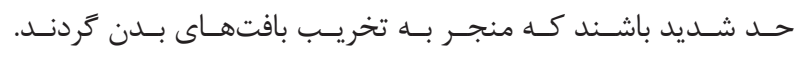

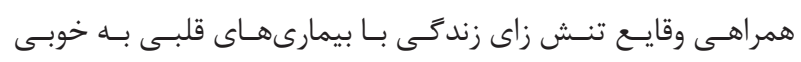




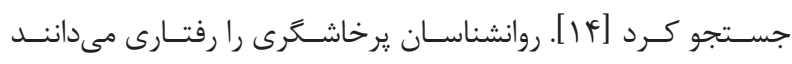

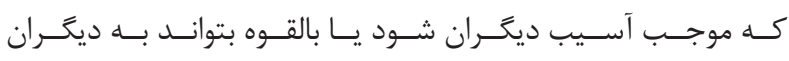

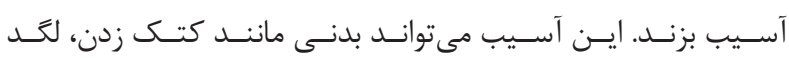

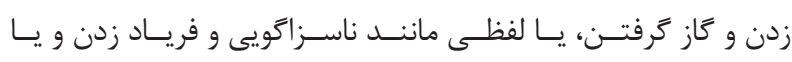

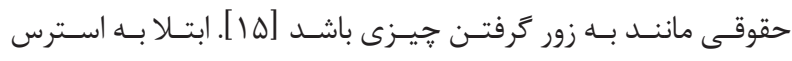

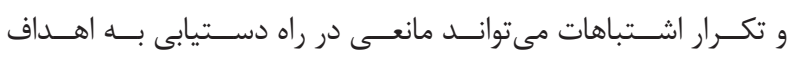

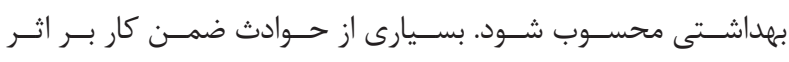

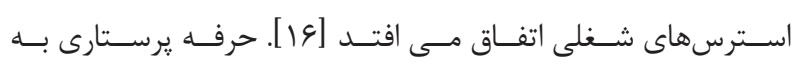

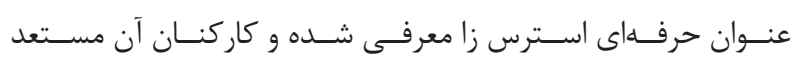

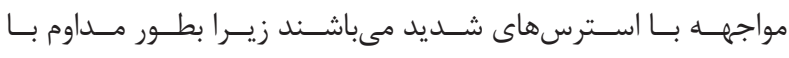

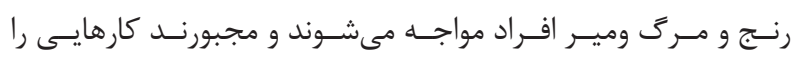

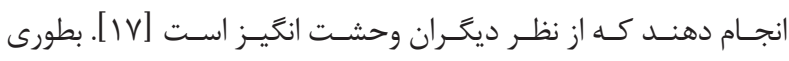

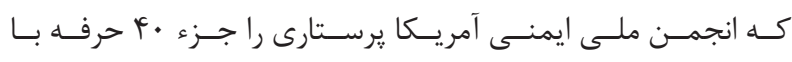

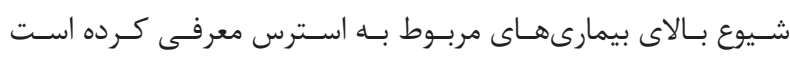

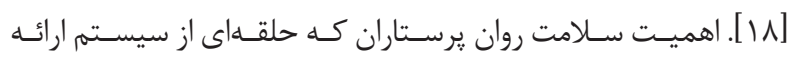
خدمـات بهداشـتى هسـتند و ضعـف ريشــينه مطالعاتـى در زمينـهـ

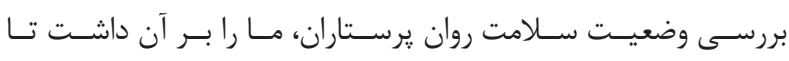

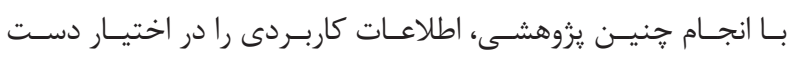

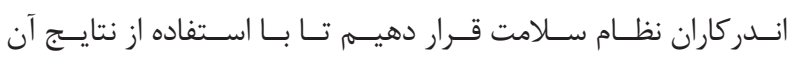

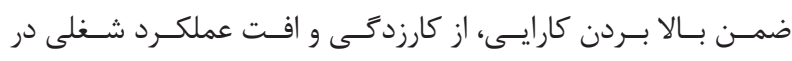

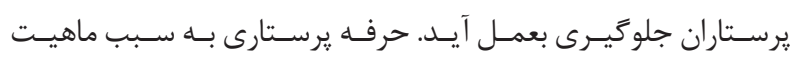

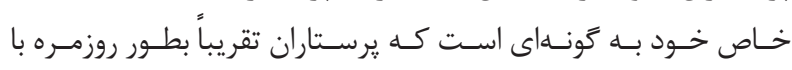

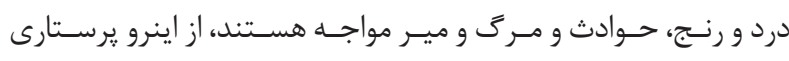

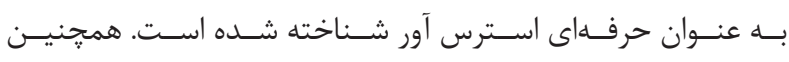

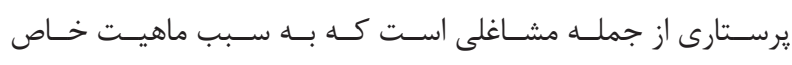

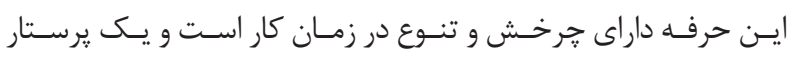

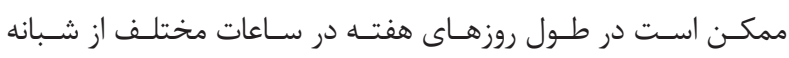

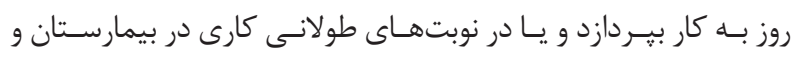

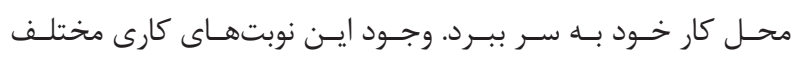

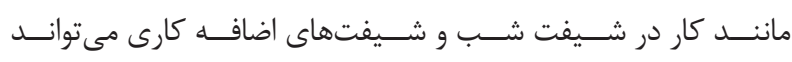

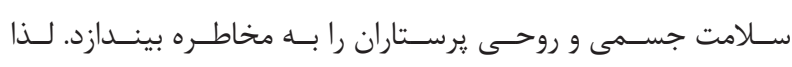

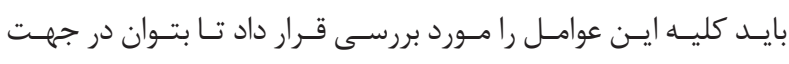

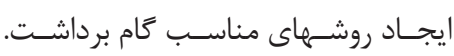

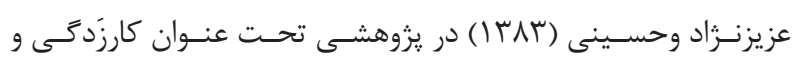

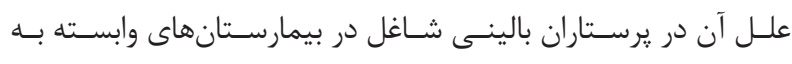

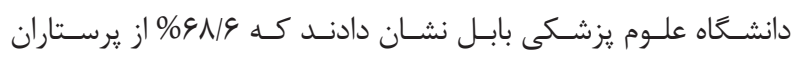

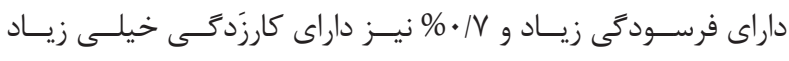

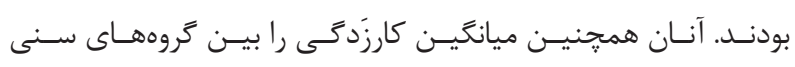

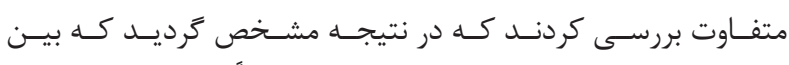

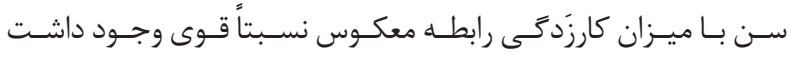

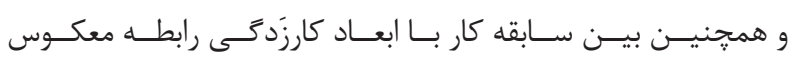

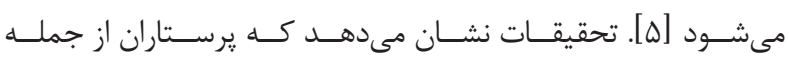

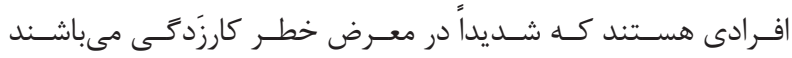

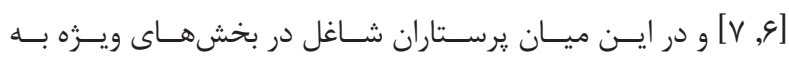

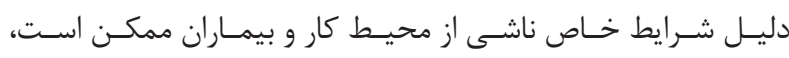

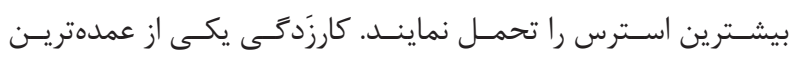

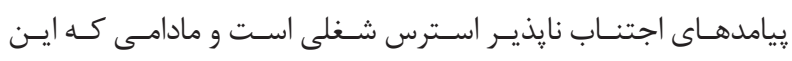

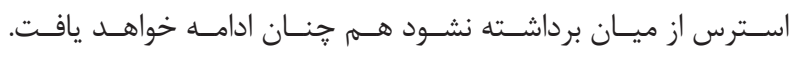

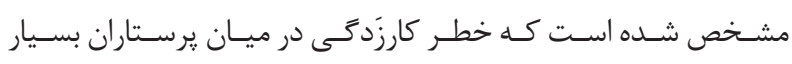

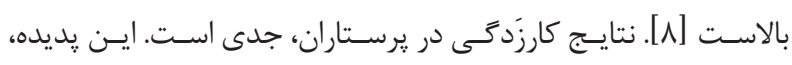

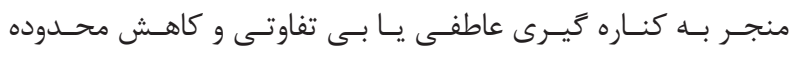

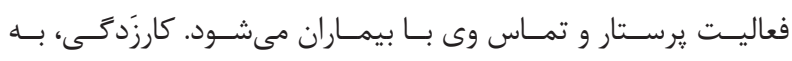

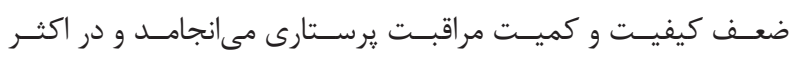

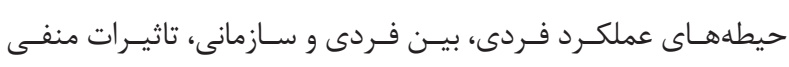

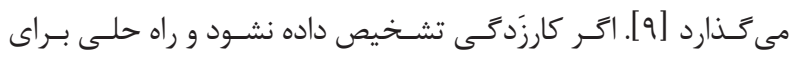

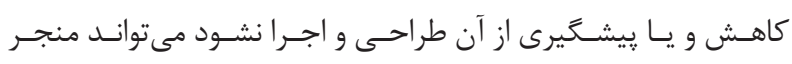

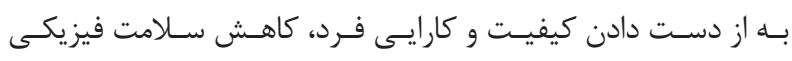

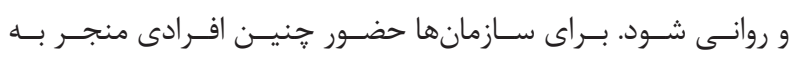

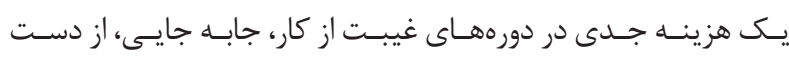

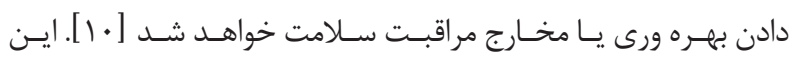

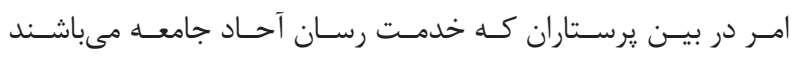

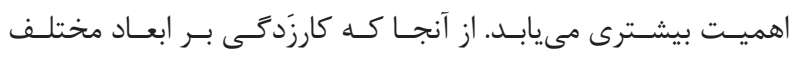

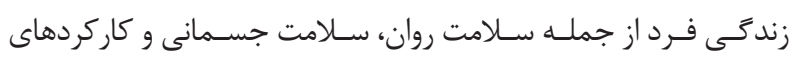

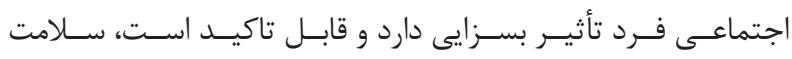

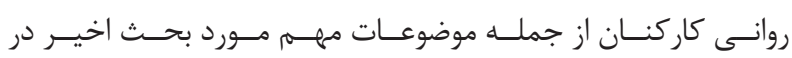

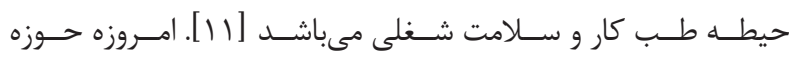

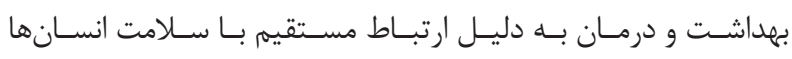

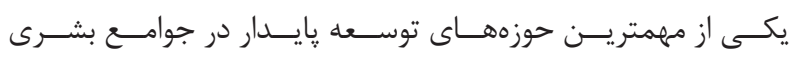

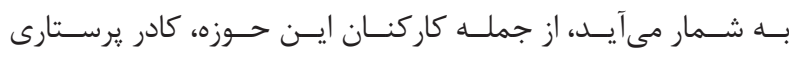

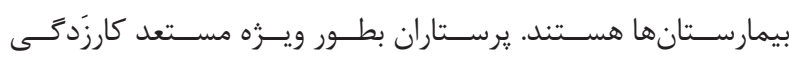

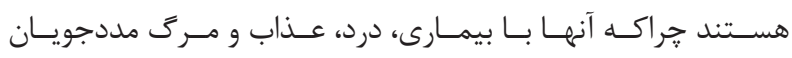

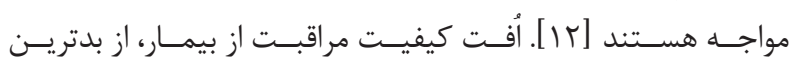

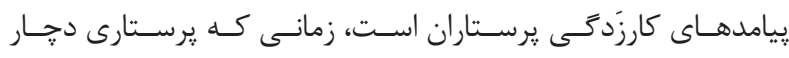

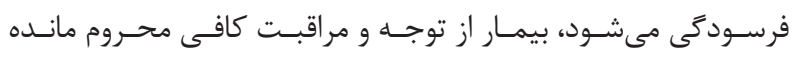

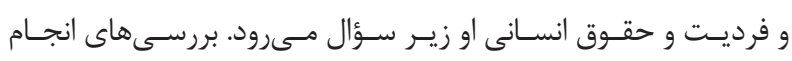

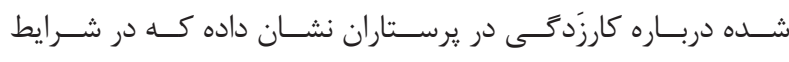

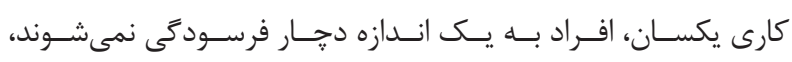

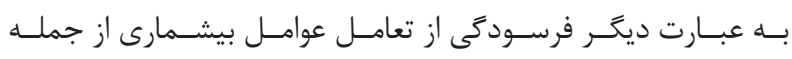

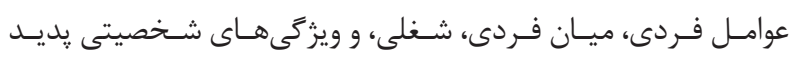

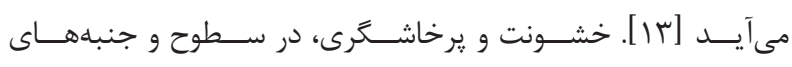

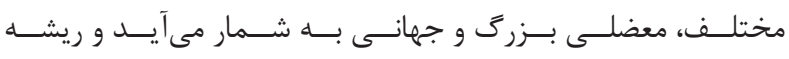

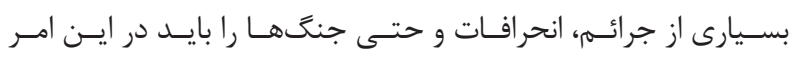




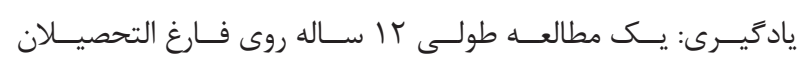

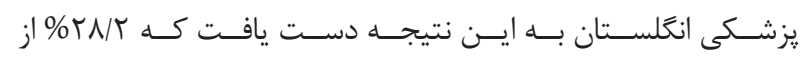

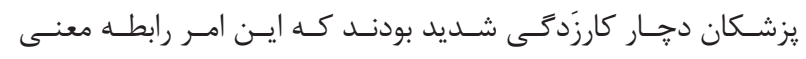

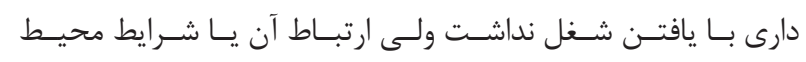

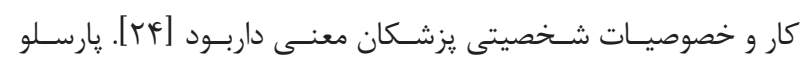

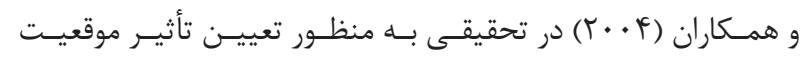

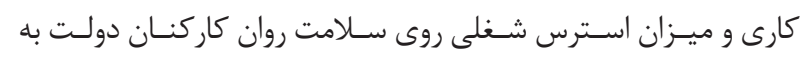

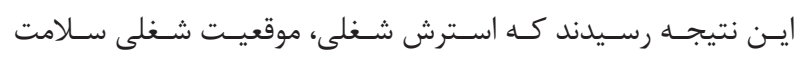

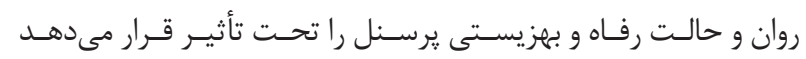

[ro]

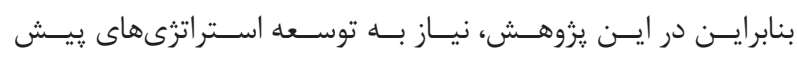

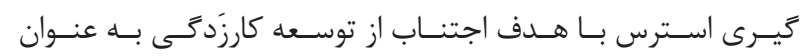

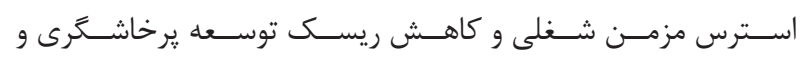

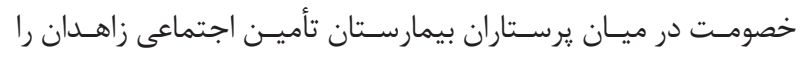

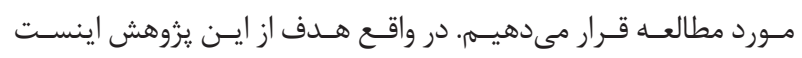

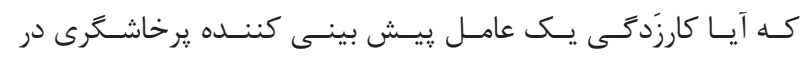

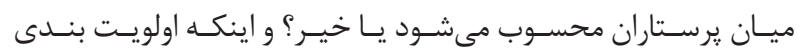

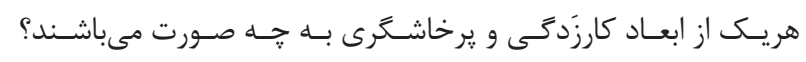

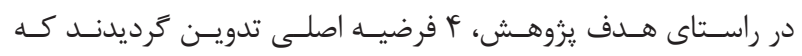

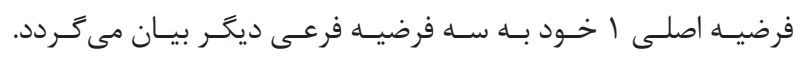

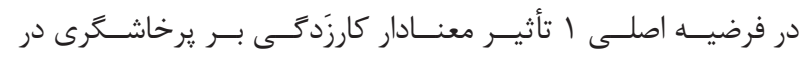

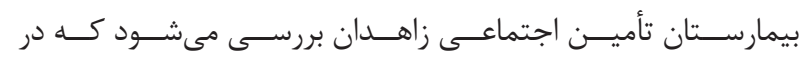

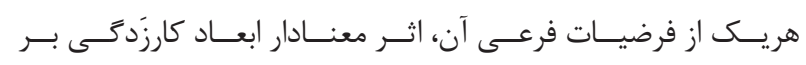

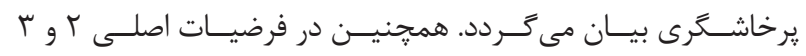

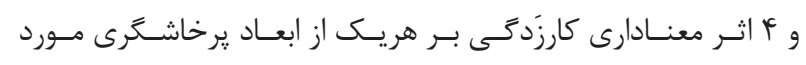

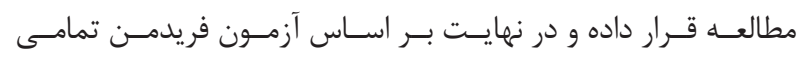

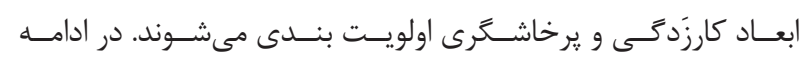

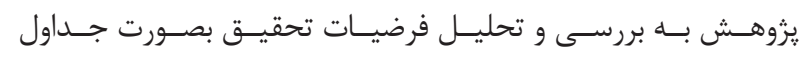

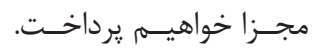

\section{روش كار}

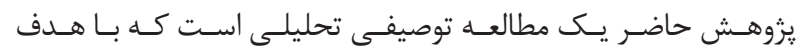

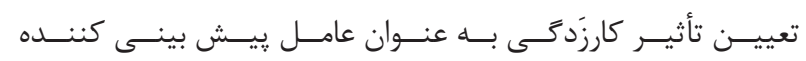

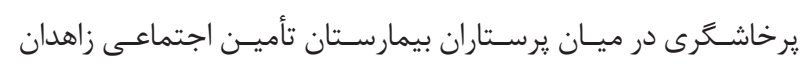

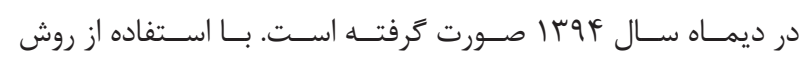

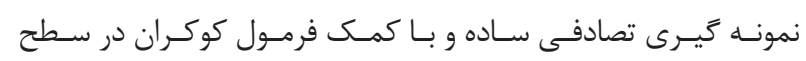

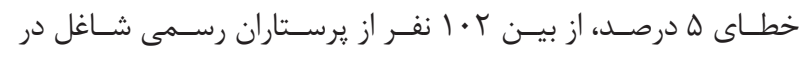

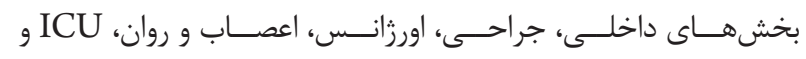

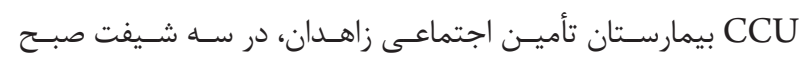

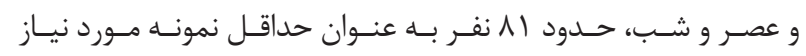

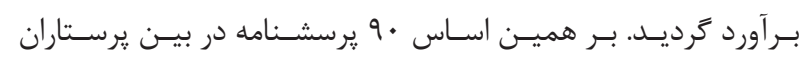

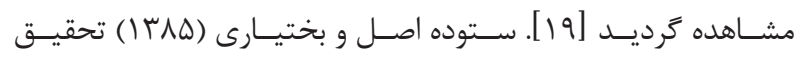

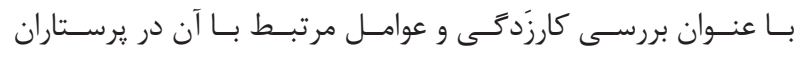

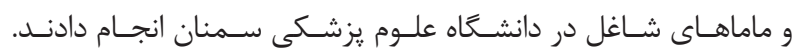

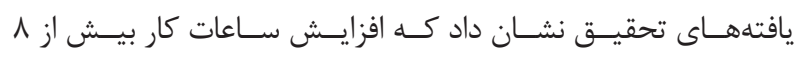

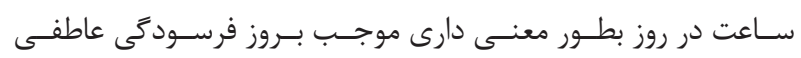

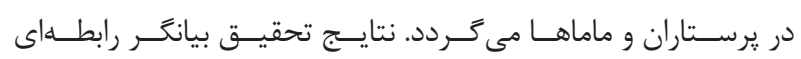

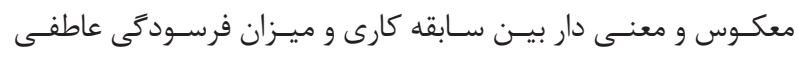

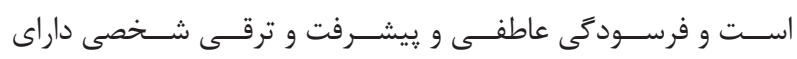

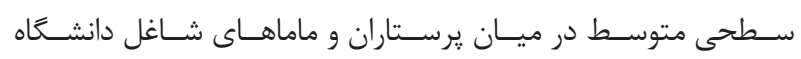

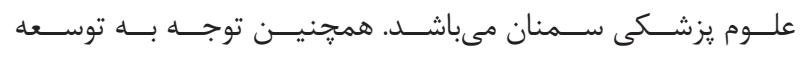

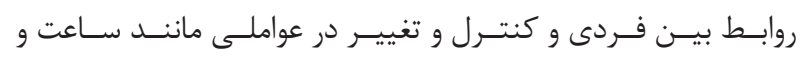

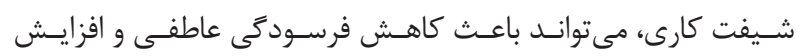

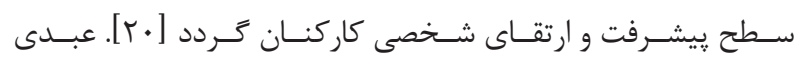

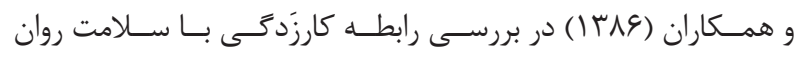

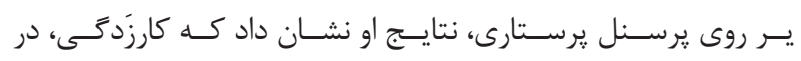

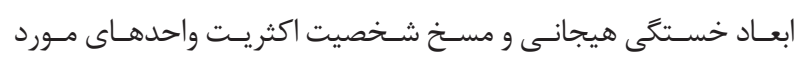

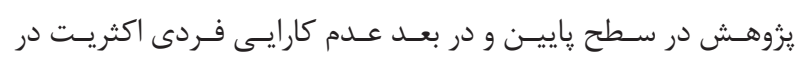

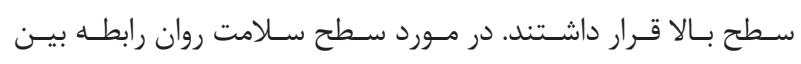

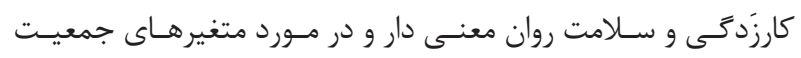

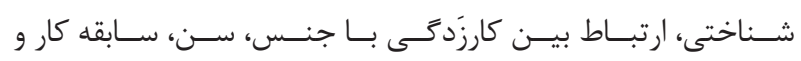

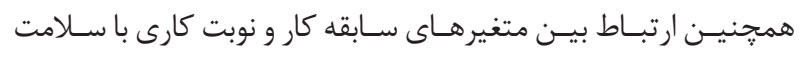

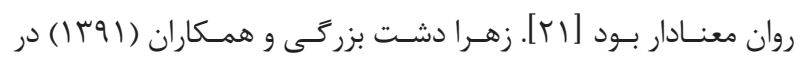

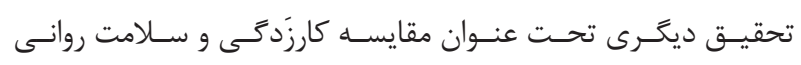

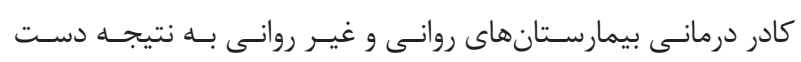

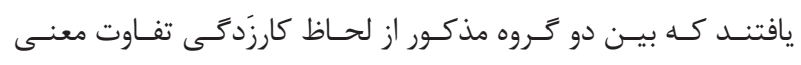

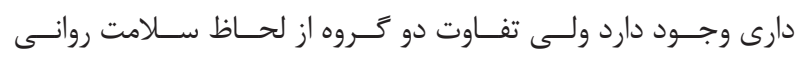

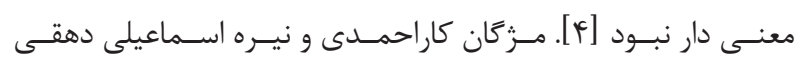

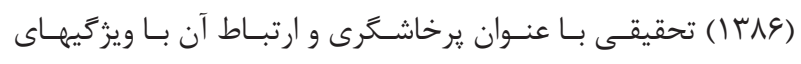

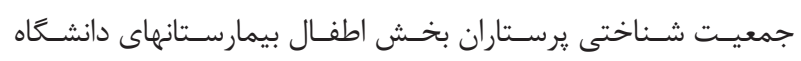

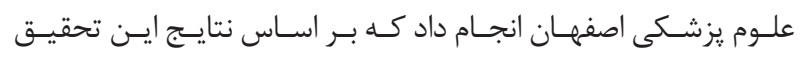

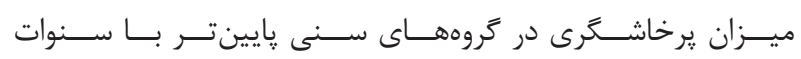

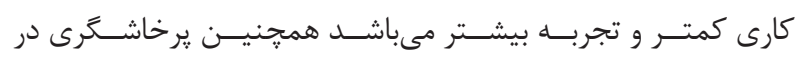

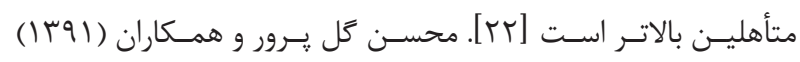

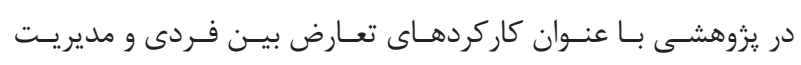

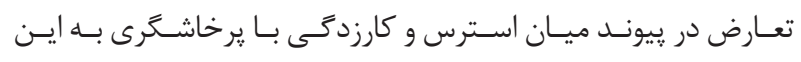

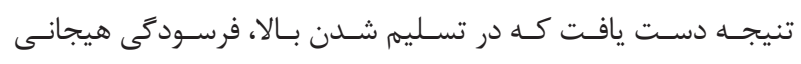

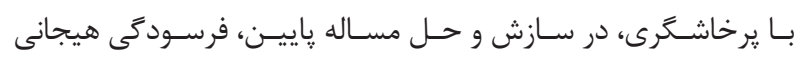

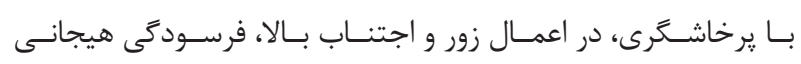

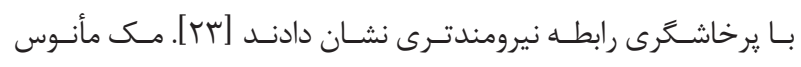

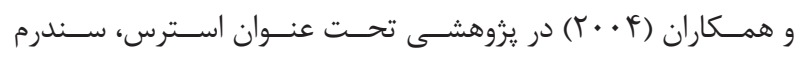

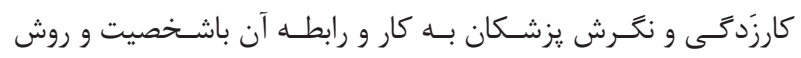




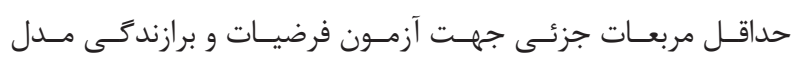

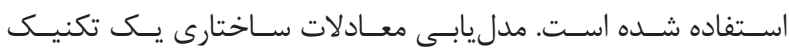

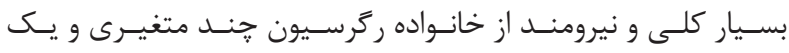

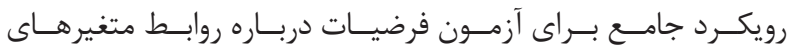

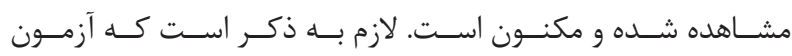

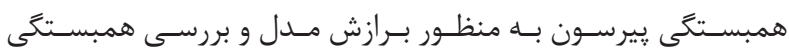

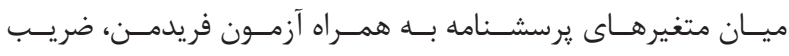

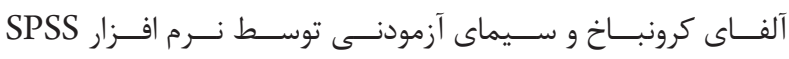

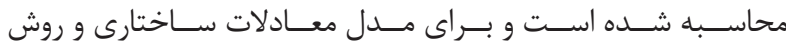

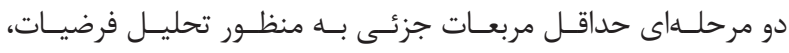

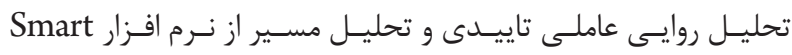

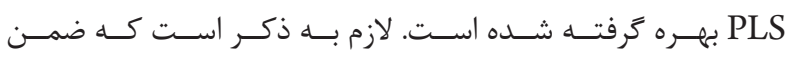

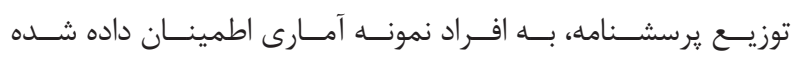

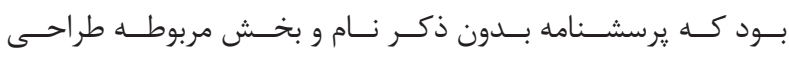

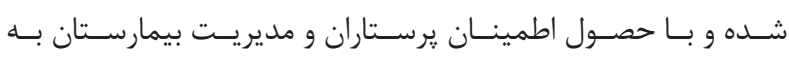

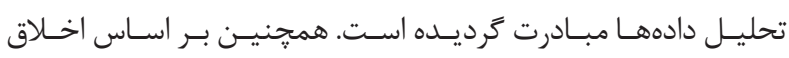

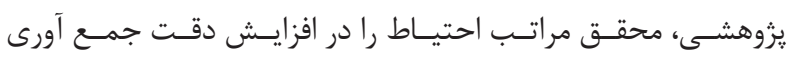

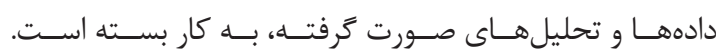

\section{يافتهها}

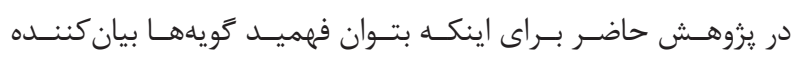

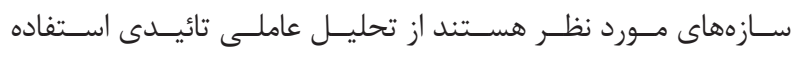

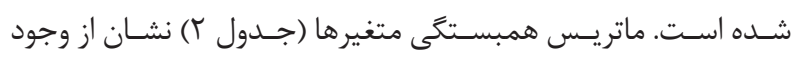

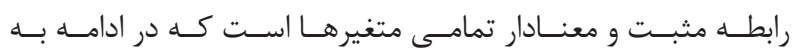

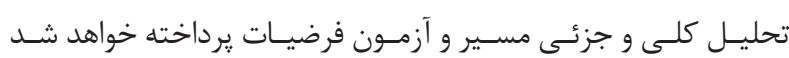

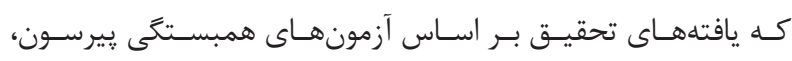

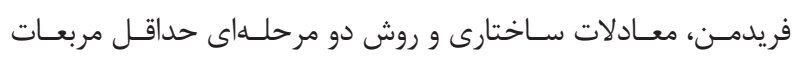

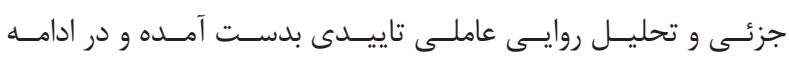

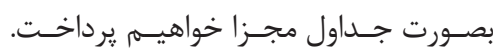

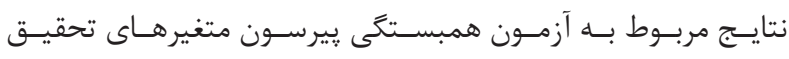

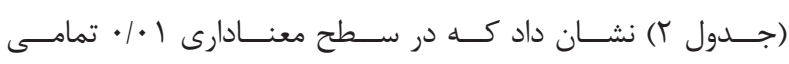

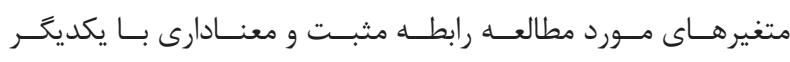

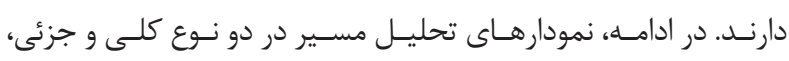

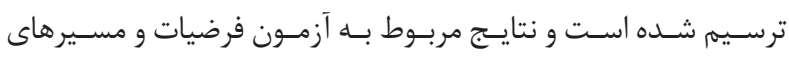

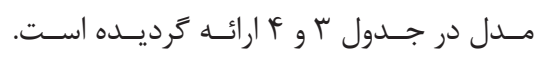

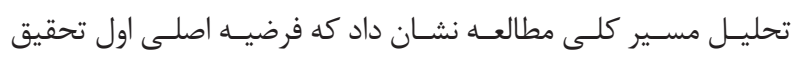

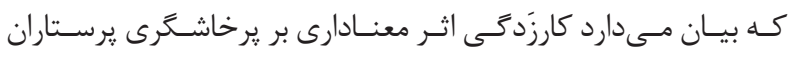

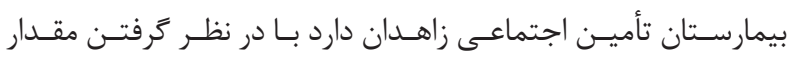

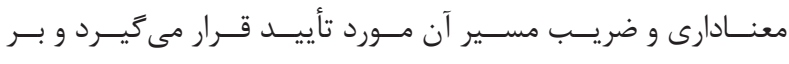

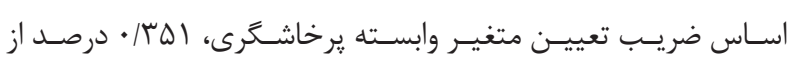

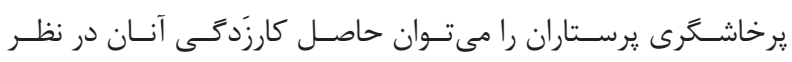

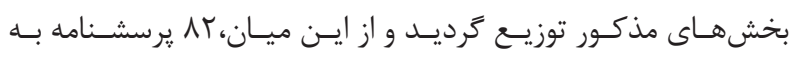

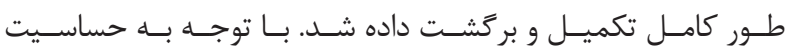

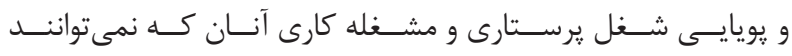

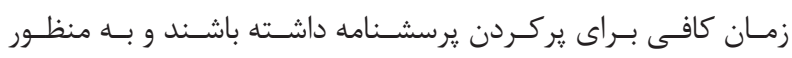

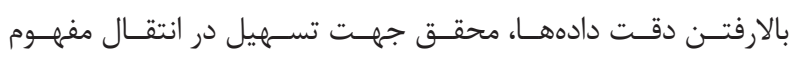

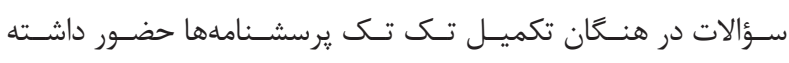

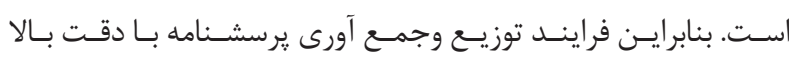

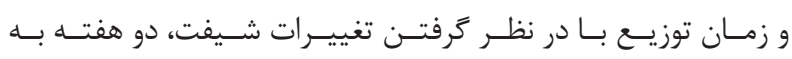

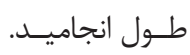

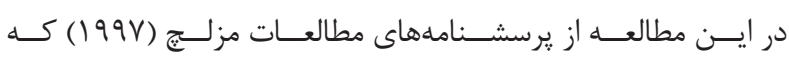

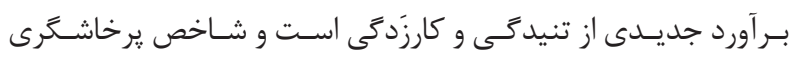

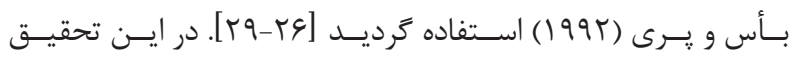

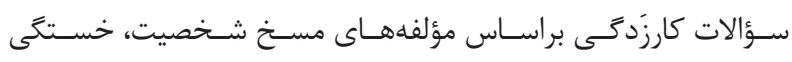

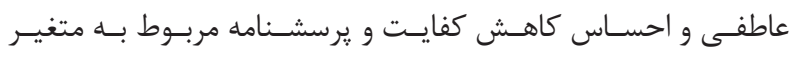

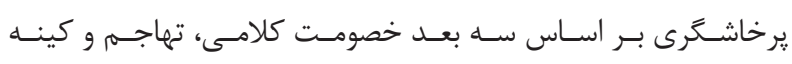

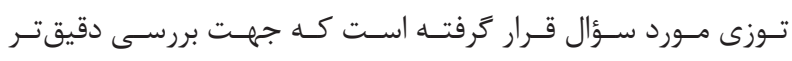

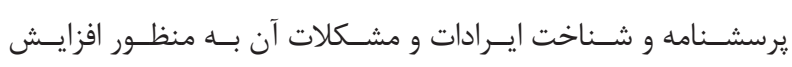

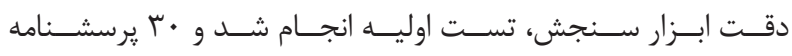

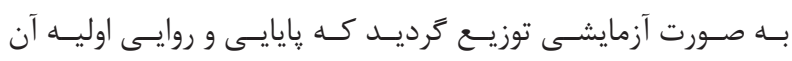

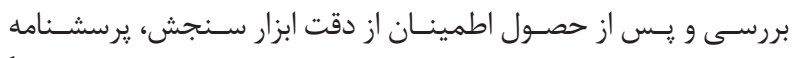

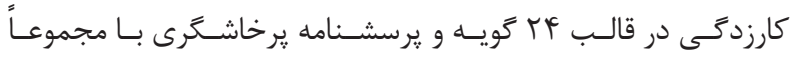

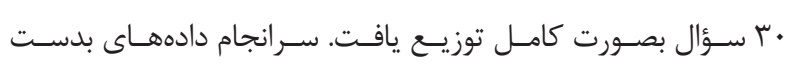

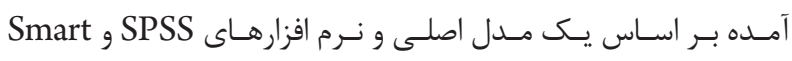

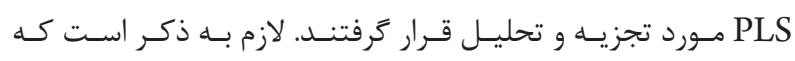

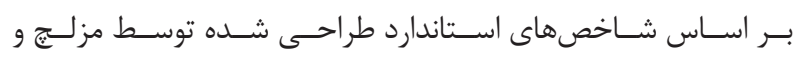

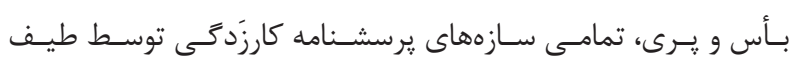

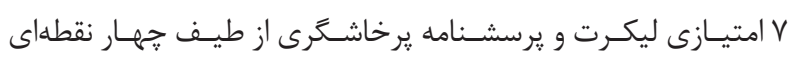

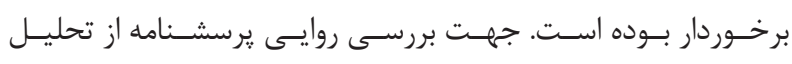

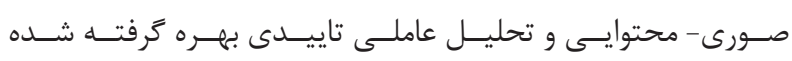

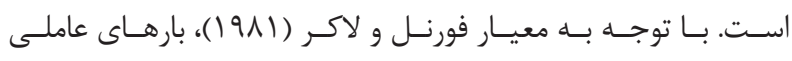

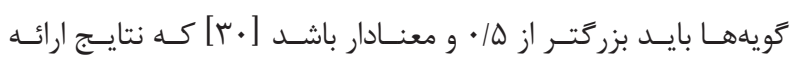

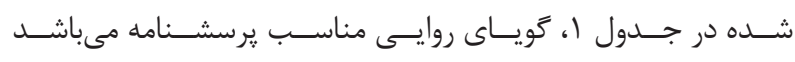

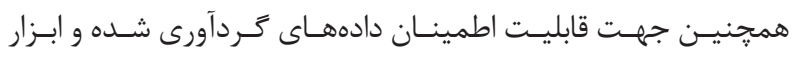

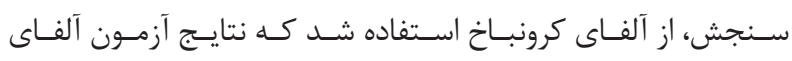

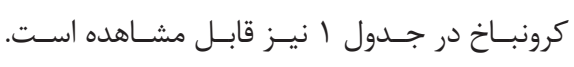

$$
r_{2}=\frac{J}{J-1}\left(1-\frac{\sum S_{j}^{2}}{S^{2}}\right)
$$

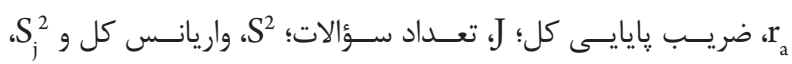
واريانسس سـؤوالات.

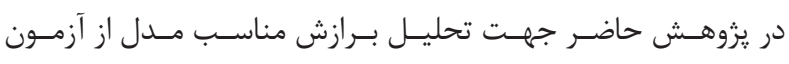

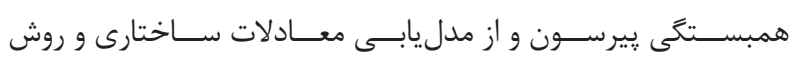




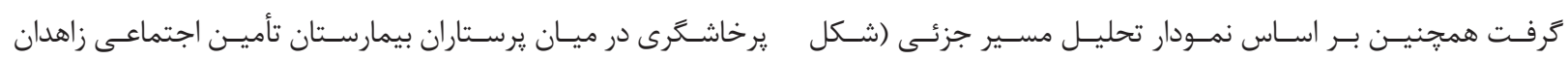

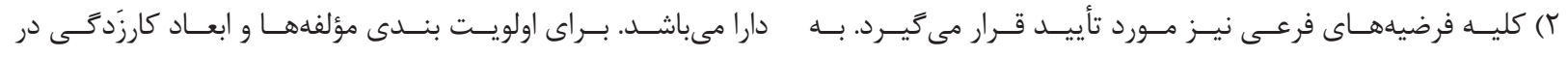

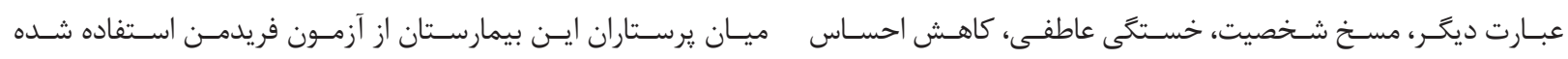

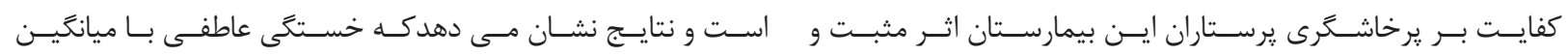

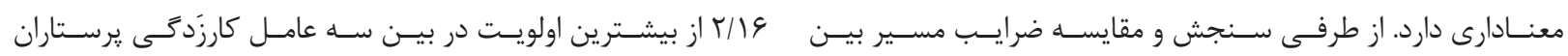

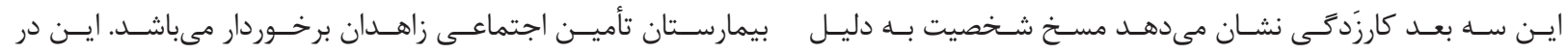

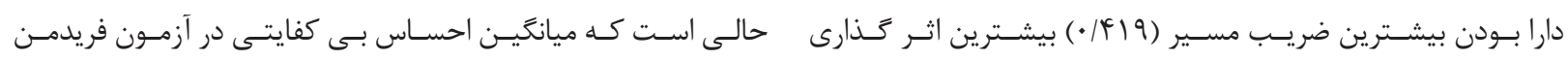

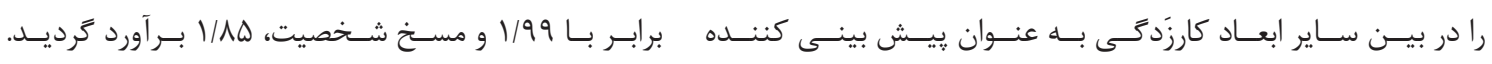

\begin{tabular}{|c|c|c|c|c|}
\hline \multicolumn{5}{|c|}{ جدول ا: توصيف آمارى، روايى و بايايى } \\
\hline ضرايب بار عاملى & آلفاى كرونباخ & ميانغين & ت تعداد سؤالات & متغيرها \\
\hline$\cdot 1949$ & - NAK & T/FYA & r. & 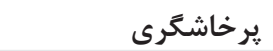 \\
\hline . & $\cdot / \Lambda \cdot 1$ & $r / v 19$ & TF & كارزَدَى \\
\hline$\cdot \mid \Delta \wedge \varphi$ & $r / 911$ & $\cdot / V I F$ & $\checkmark$ & مسخ شخصيت \\
\hline $.94 \pi$ & $\cdot / \mathrm{d} \cdot \mathrm{r}$ & $r / \Lambda 1 \Delta$ & 4 & خستكى عاطفى \\
\hline$\cdot \mid 9 M 1$ & $\cdot / V \Delta \Delta$ & $r / v r \cdot$ & 11 & كاهش احساس كفايت \\
\hline
\end{tabular}

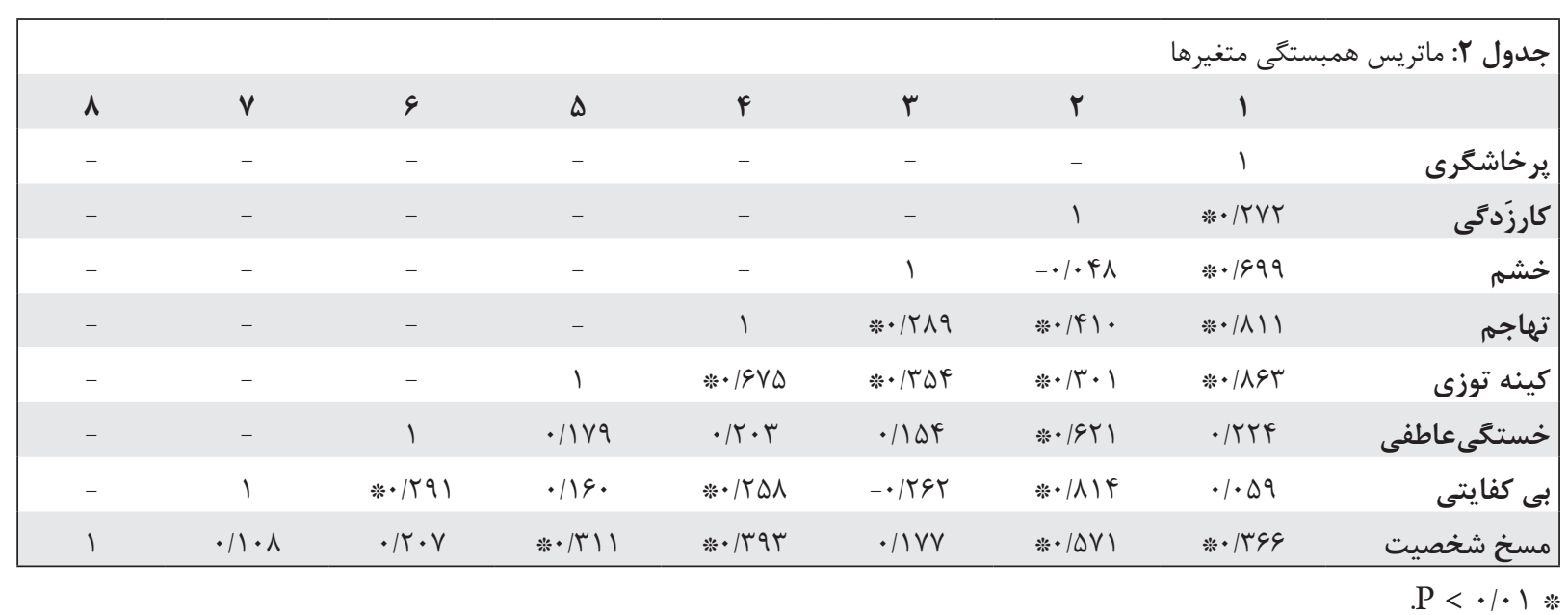

\begin{tabular}{|c|c|c|c|c|c|}
\hline \multirow[b]{2}{*}{ مقدار معنادارى } & \multirow[b]{2}{*}{ ضر يب تعيين (\%) } & \multirow[b]{2}{*}{ نتايج } & \multicolumn{3}{|c|}{ جدول با: نتايج تحليل فرضيه اصلى ا و فرضيات فرعى آن } \\
\hline & & & همبستكى & t & فرضيات اصلى و فرعى 1 \\
\hline.$/ . .1$ & $\cdot / r \Delta 1$ & تأييد & g/DAF & .199. & فرضيه ا: كارزَدَى بر برخاشعَىى \\
\hline$\cdot 1 \cdot .1$ & $\cdot /$ TVG & تأييد & $1 / 910$ &.$/ 419$ & فرضيه |-1: مسخ شخصيت بر برخاشكَى \\
\hline .1 .1 & $\cdot r v q$ & تأييد & t/Frq &.$/ 119$ & فرضيه ا-r: خستَّى عاطفى بر يرخاشكَىى \\
\hline .1 .1 & $\cdot \pi \vee q$ & تأييد & $r / 4 q$. & $\cdot / K M F$ & فرضيه ا-ץ: احساس بى كفايتى بر يرخاشكَرى \\
\hline
\end{tabular}

\begin{tabular}{|c|c|c|c|c|c|}
\hline \multirow[b]{2}{*}{ مقدار معنادارى } & \multirow[b]{2}{*}{ ضريب تعيين (\%) } & \multirow[b]{2}{*}{ نتايج } & \multirow[b]{2}{*}{ همبستكى } & \multicolumn{2}{|c|}{ جدول f: نتايج تحليل فرضيه اصلى r، بو F } \\
\hline & & & & t & فرضيات \\
\hline $.1 \cdot .1$ & $\cdot / r \Delta q$ & تأييد & $-g / V \wedge F$ & -.1099 & فرضيه ז: كارزَدَى بر خشم \\
\hline$\cdot 1 . .1$ & $\cdot / 1 \wedge \vee$ & تأييد & F/Tq৭ & 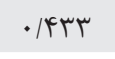 & فرضيه rا: كارزدَّى بر تهاجم \\
\hline.$\cdots$ &.$/ 1 \mu$ & تأييد & $r / 499$ &. mak & فرضيه F: كارزدَّى بر كينه توزى \\
\hline
\end{tabular}




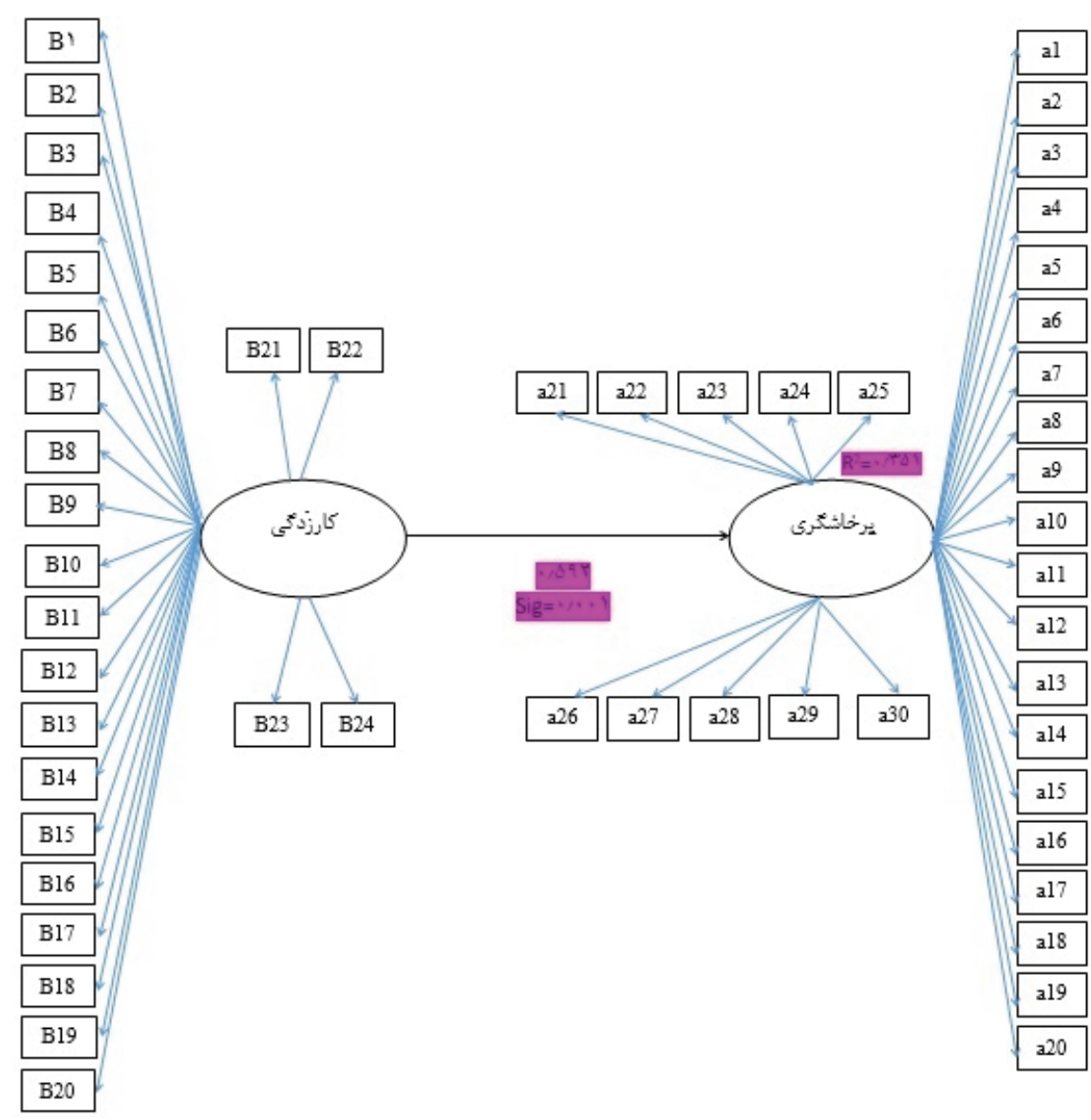

تصوير ا: تحليل مسير كلى

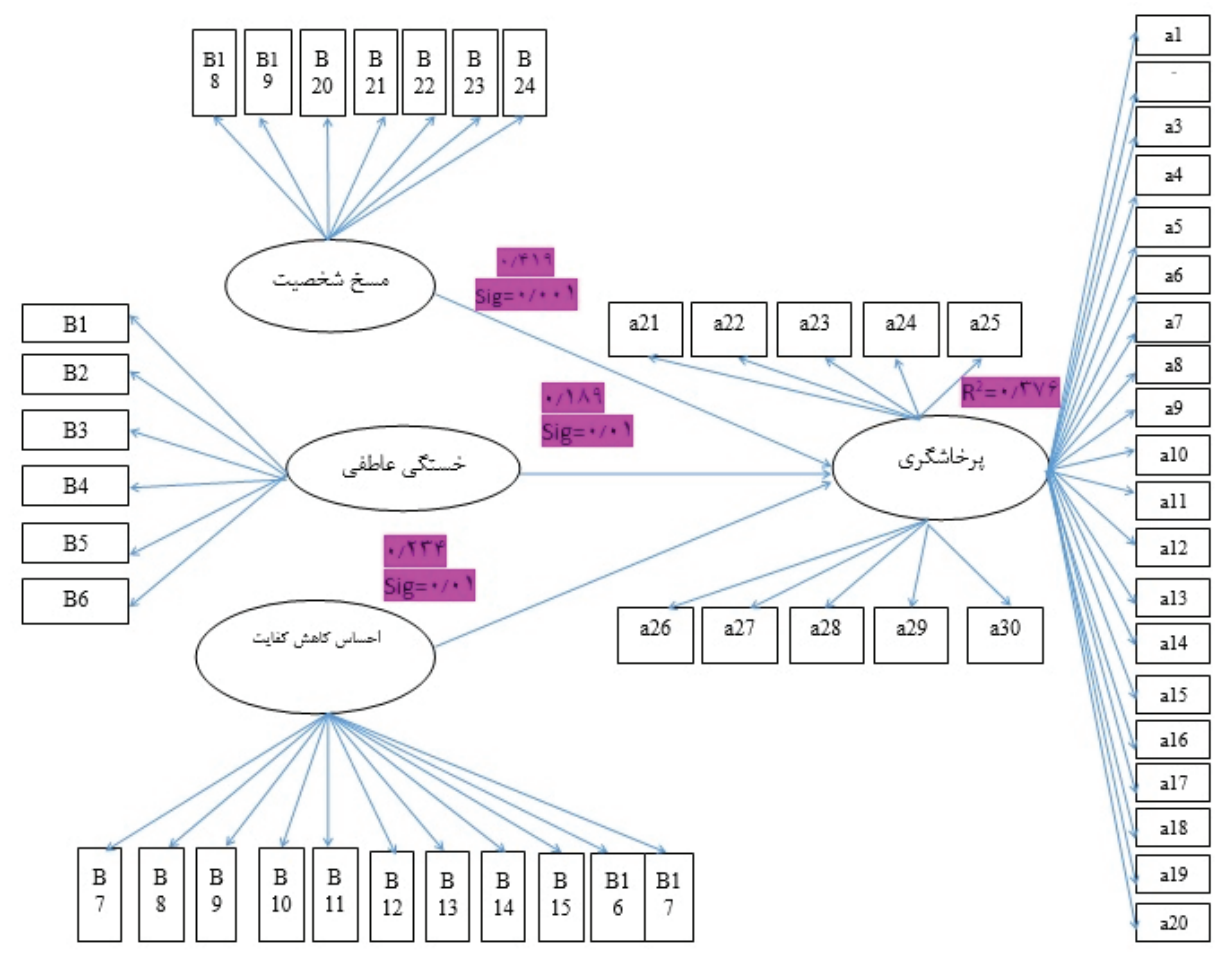




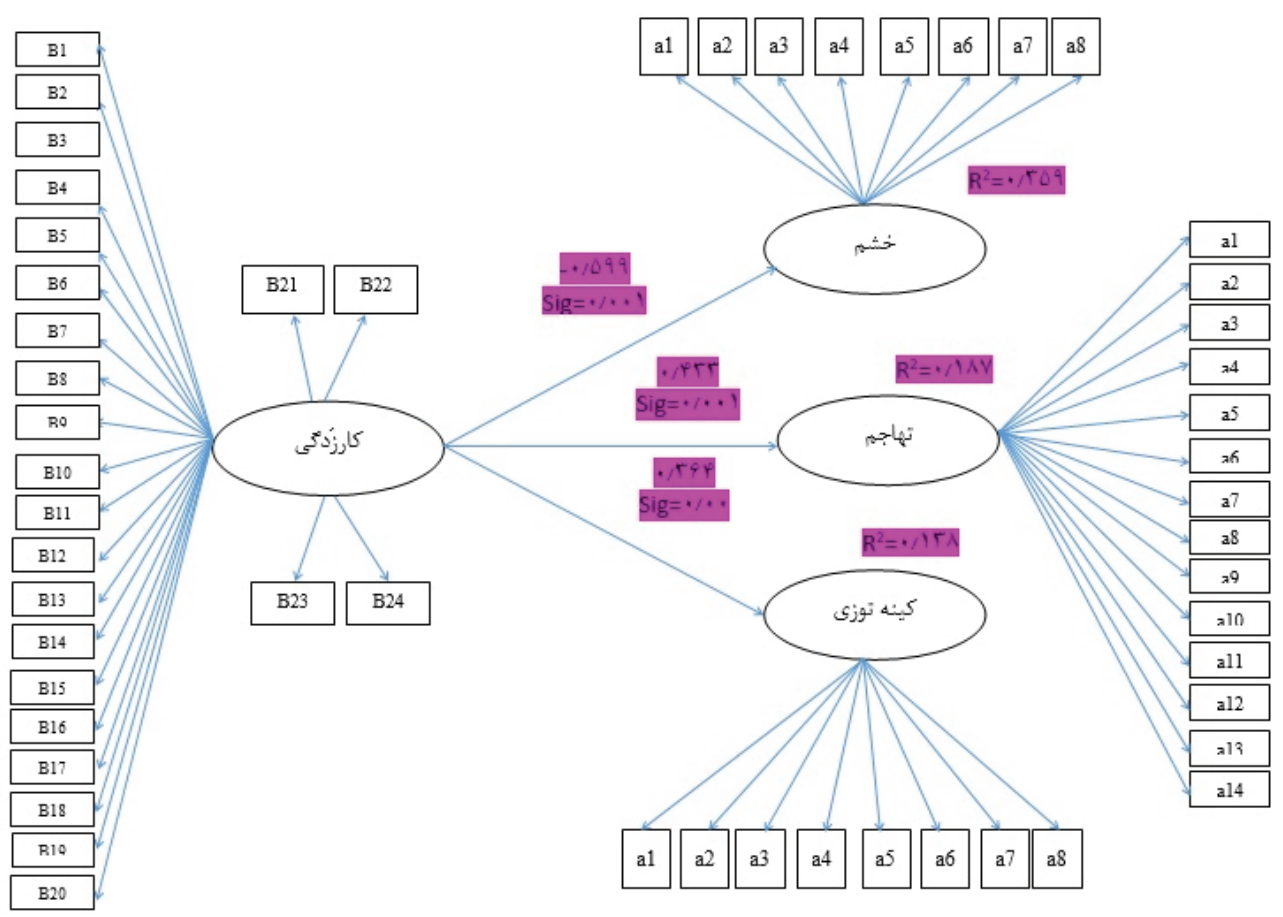

تصوير ץ: تحليل مسير جزئى(ابعاد يرخاشكرى)

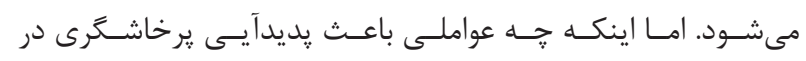

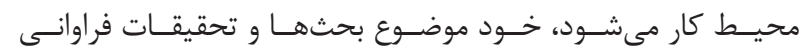

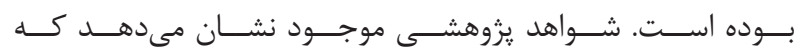

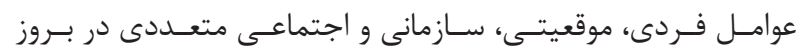

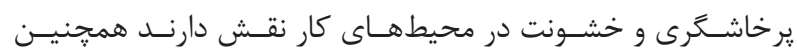

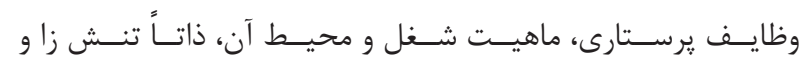

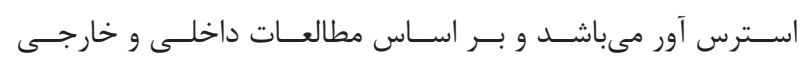

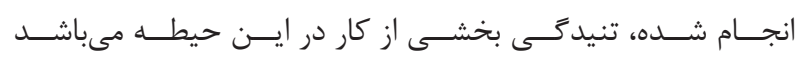

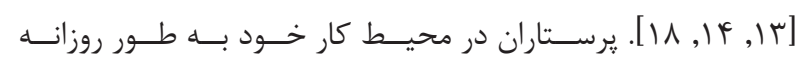

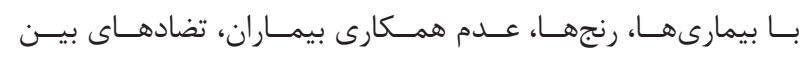

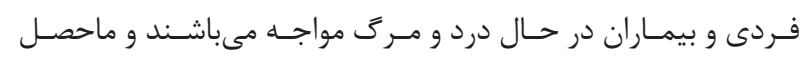

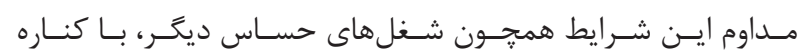

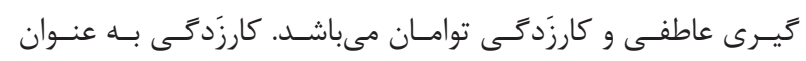

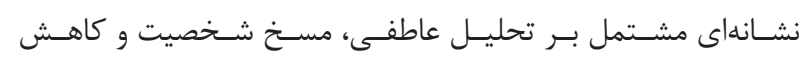

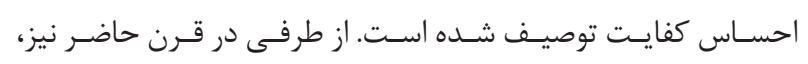

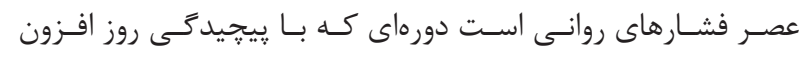

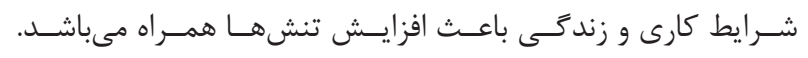

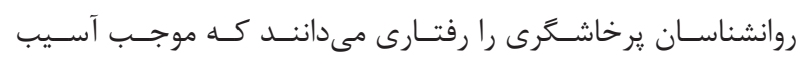

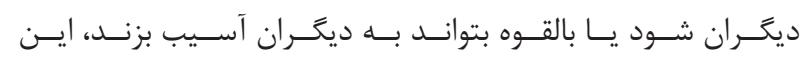

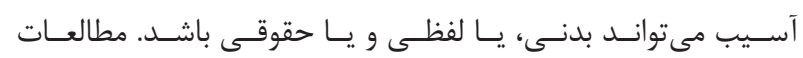

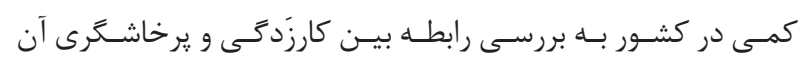

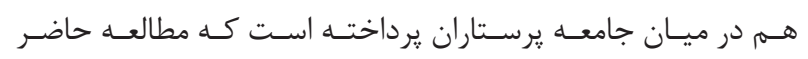

بــر اسـاس تحليـل دادههـا و بــا توجـهـ بــه فرضيـات عنــوان شـده،

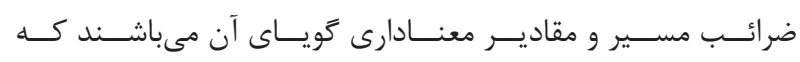

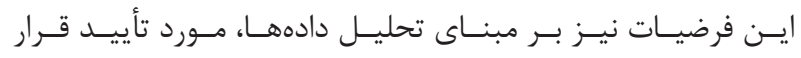

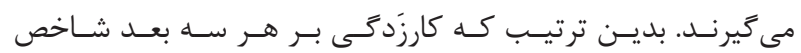
يرخاشــرى اثــر مثبـت معنــادارى دارد.

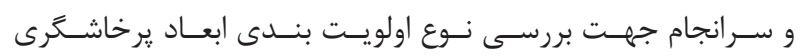

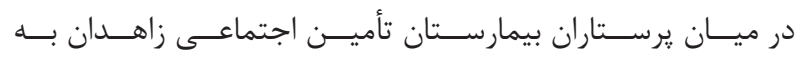

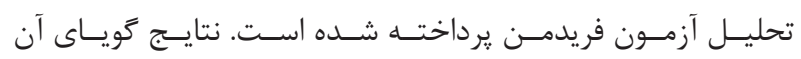

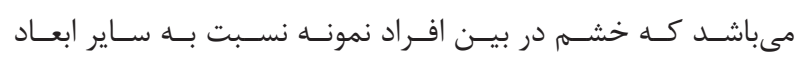

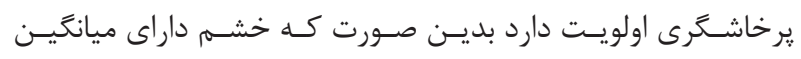
T/VF

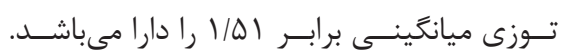

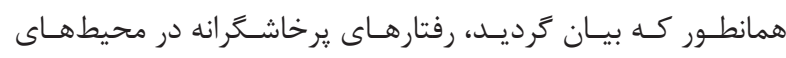

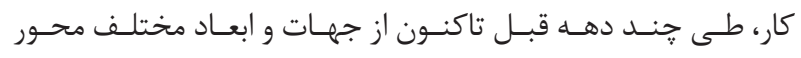

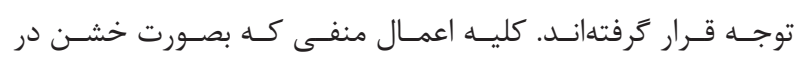

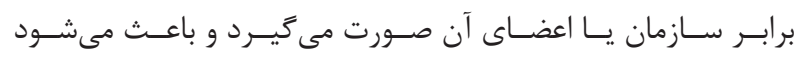

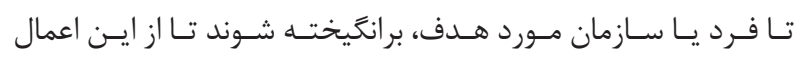

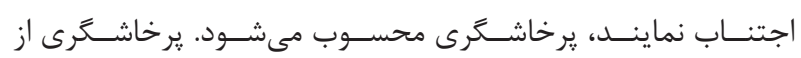

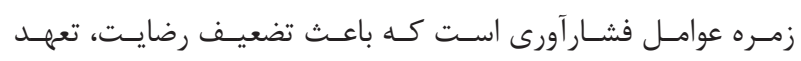

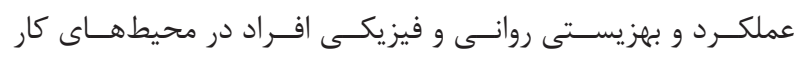


مسـتقيم داشـته اسـت و ايــن موضـوع همسـو بــا فرضيـات تأييـد

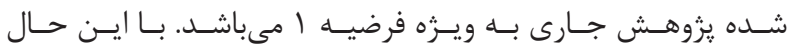

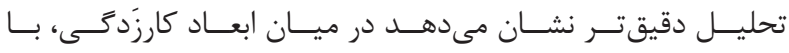

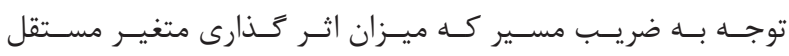

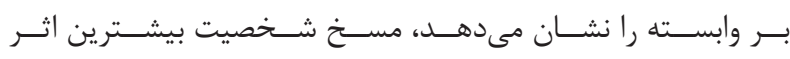

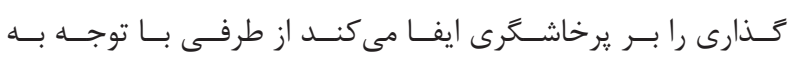

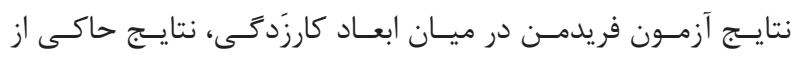

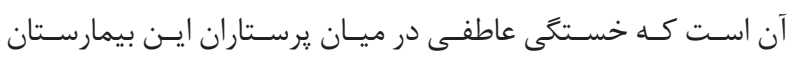

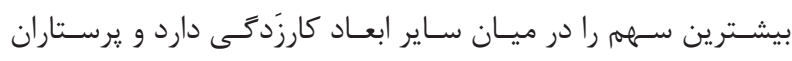

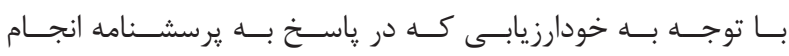

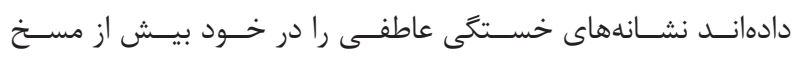

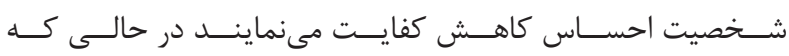

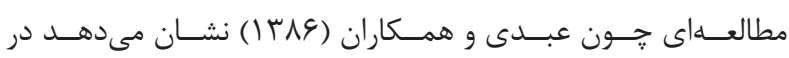

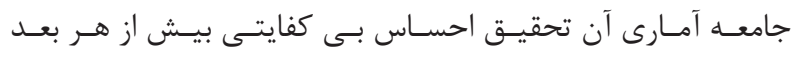

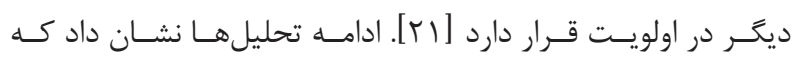

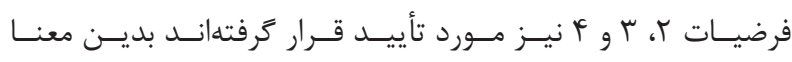

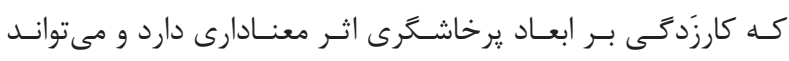

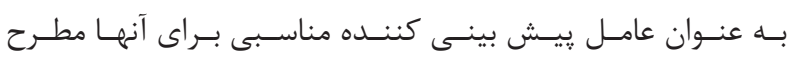

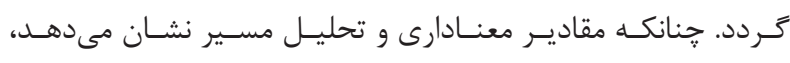

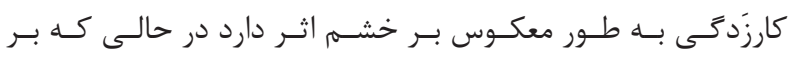

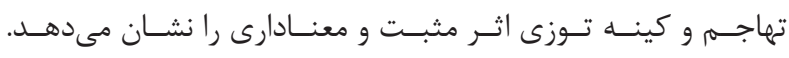

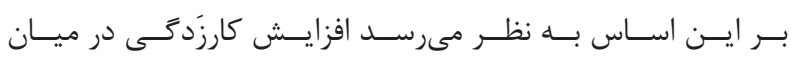

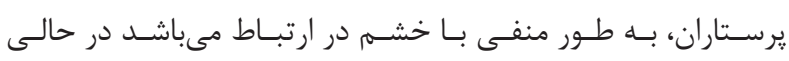

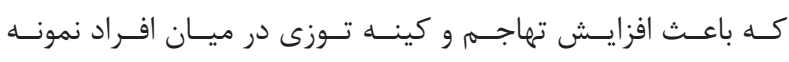

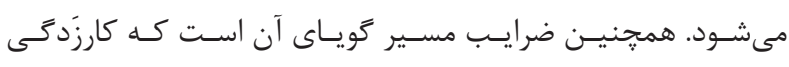

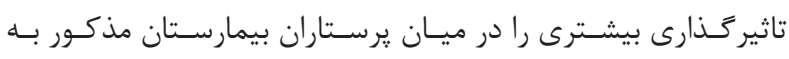

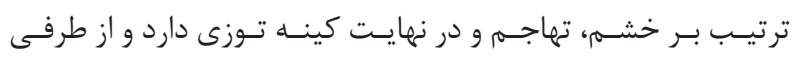

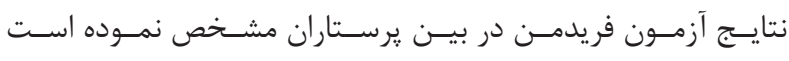

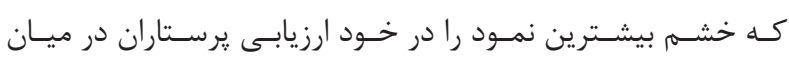

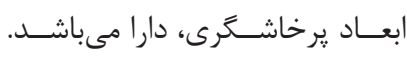

نتيجه كيرى

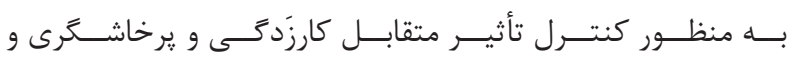

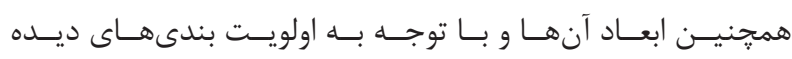

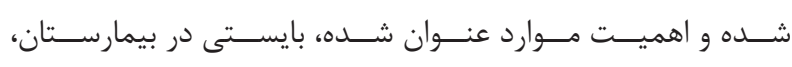

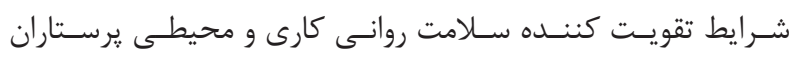

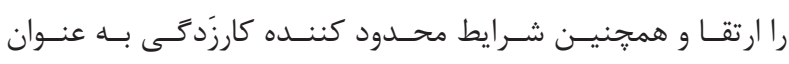

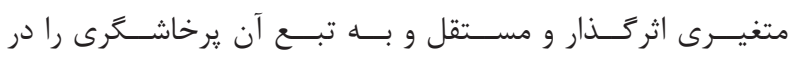

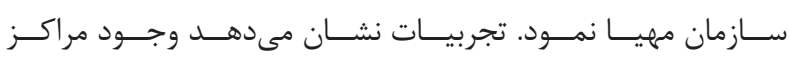

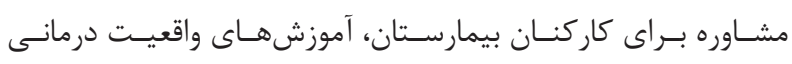

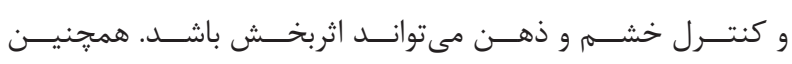

بــا توجـهـهـ بـهـ اهميـت ايسن دو وضعيـت روحسى و روانسى كـهـ ماحصـل

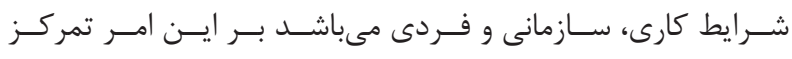

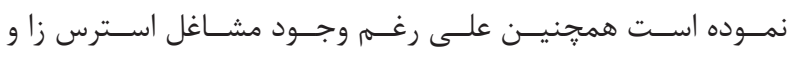

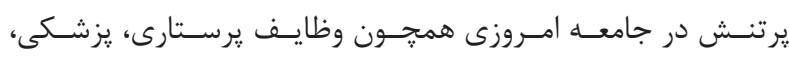

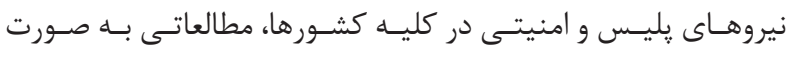

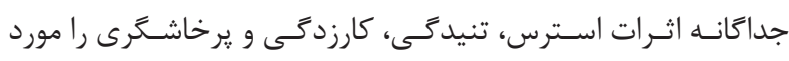

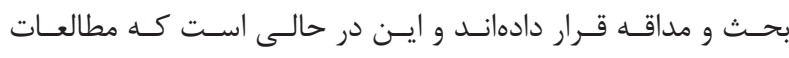

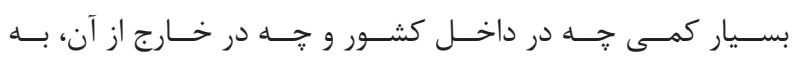

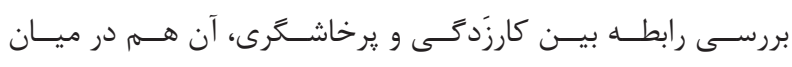

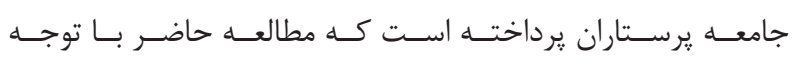

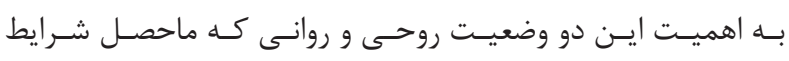

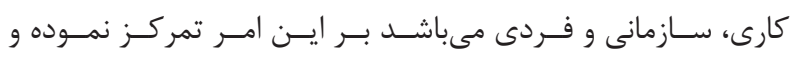

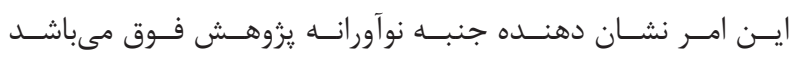

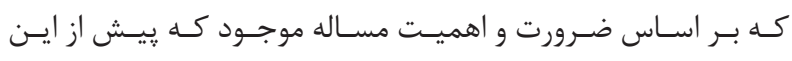

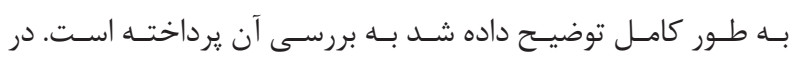

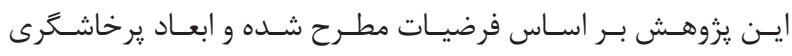

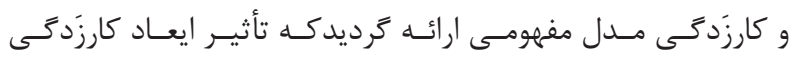

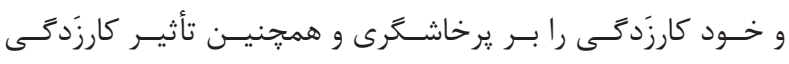

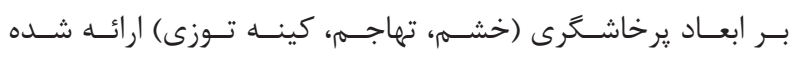

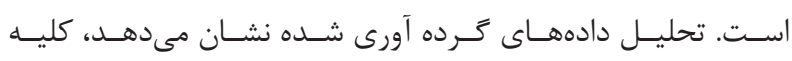

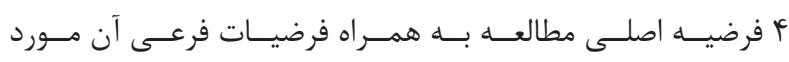

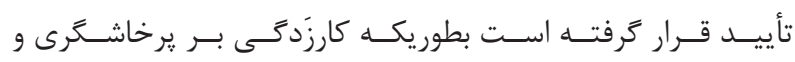

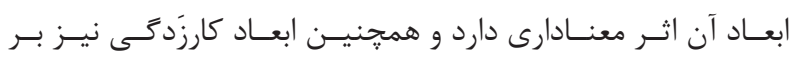

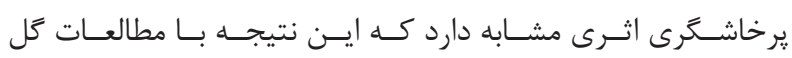

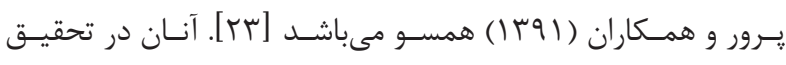

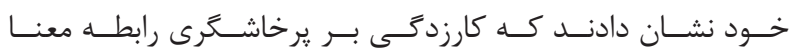

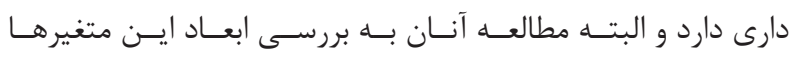

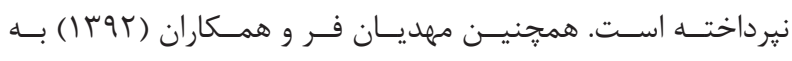

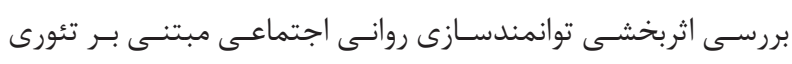

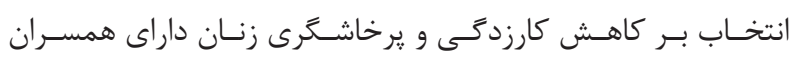

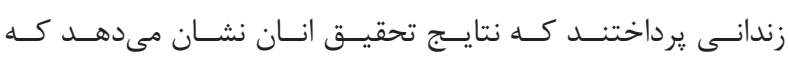

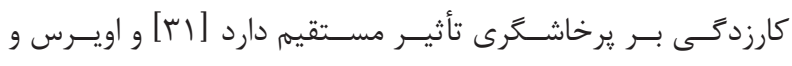

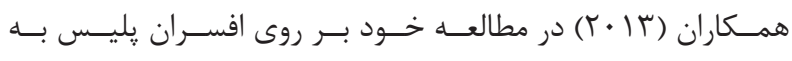

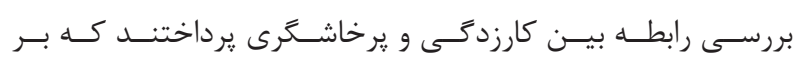

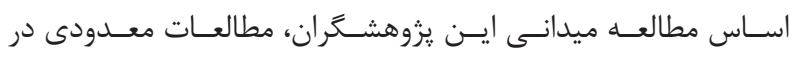

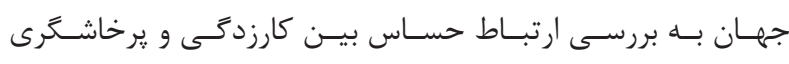

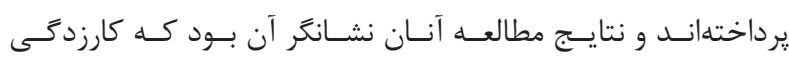

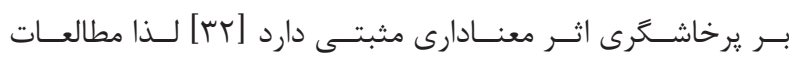

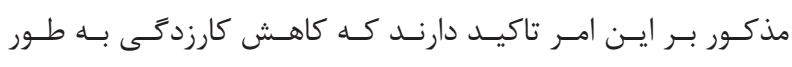

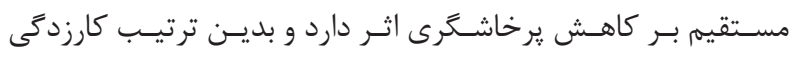

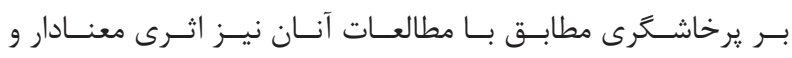




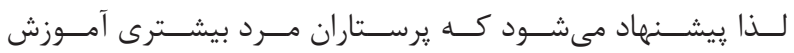

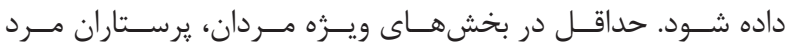

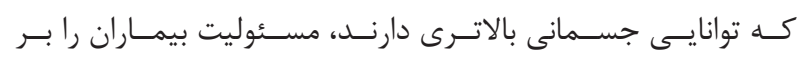

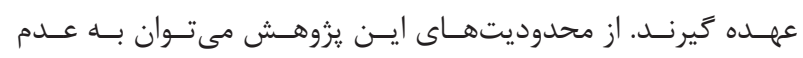

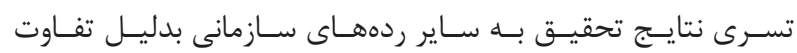

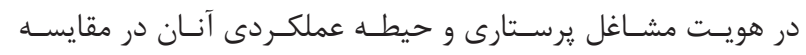

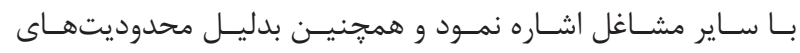

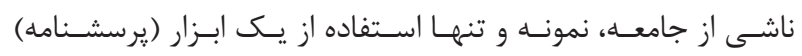

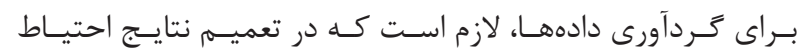

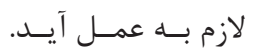

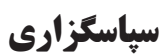

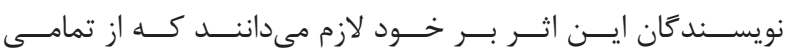

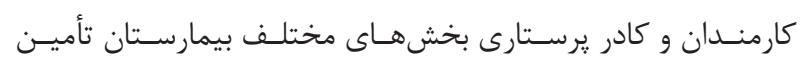

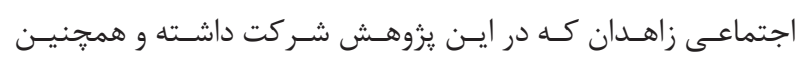

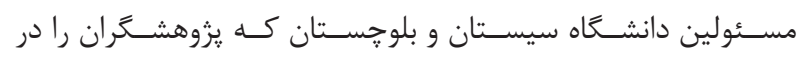
ايسن امـر يـارى نمودنــ، تشـكر و قدردانـى نماينـد.

تضاد منافع

در اين مطالعه تضاد منافع وجود ندارد.

\section{REFERENCES}

1. Sardarpour Goudarzi SH, Zarandi A. [Mental health and Fasting in Ramadan]. Iran J Psychiatr Clin Psychol. 2003;8(2):26-32.

2. Safair S, Fattahzadeh AA. [Gbbal health report in 2003,The Cultural institute of great Ibnesine]. Ministry Treat Med Troun. 2003:12-5.

3. Tsutsumi A, Kayaba K, Nagami M, Akiko M, Kawano Y, Odagiri Y, et al. The effort-reward imbalance model: experience in Japanese working population. J Occupat Health. 2002;44(6):398-407. DOI: 10.1539/joh.44.398

4. Dashte bozorgi Z, Amirie majd M, Alizadeh M, Alipoor khodadadi $\mathrm{SH}$, Abafat H. [Comparison of burnout and mental health medical staff Psychiatric and non-psychiatric hospitals]. J Soci Psychol Find Psychol. 2012;24(7):113-29.

5. Schoufeli W, Bunk BP. Burnout: An overview of 25 years of research andtheorizing. In: Schabracq MJ, Winnubst JAM, Cooper CC, editors. The handbook of work and health psychology. 43. West Sussex, England2003. p. 383-429.

6. Momeni H, Salehi A, Seraji A. [Burnout in nurses in the care and education of medical science]. J Arak Univ Med Sci. 2008;12(4):12313.

7. Khamrnia M, Torani S, Mohammadi R. [The effect of social capital on burnout in nurses in Hasheminejad hospital]. J Hormozgan Univ Med Sci. 2010;15(3):217-09.

8. Piko BF. Burnout, role conflict, job satisfaction and psychosocial health among Hungarian health care staff: a questionnaire survey. Int J Nurs Stud. 2006;43(3):311-8. DOI: 10.1016/j.ijnurstu.2005.05.003 PMID: 15964005

9. Rafii F, Oskouie F, Nikravesh M. Factors involved in nurses' responses to burnout: a grounded theory study. BMC Nurs. 2004;3(1):6. DOI: 10.1186/1472-6955-3-6 PMID: 15541180

10. Amarjit SG, Alan BF, Mickey S. Mitigating stress and burnout by implementing transformational-leadership. Int $\mathrm{J}$ Contemporary Hospital Manage. 2006;18(6):469-81. DOI: $10.1108 / 09596110610681511$

11. Kohpayezadeh J, Agilinejad M, Mokamelkhah E, Golabadi M.
بررسـى تجربيـات بيمارسـتانهاى موفـق سـاير كشـورها در مهــار

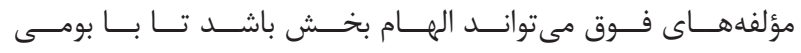

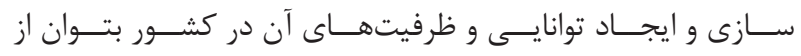

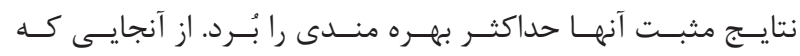

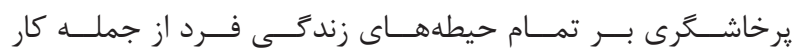

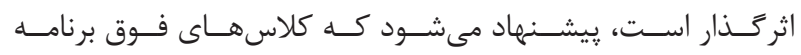

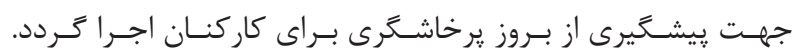

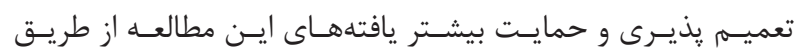

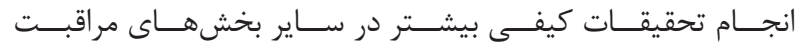

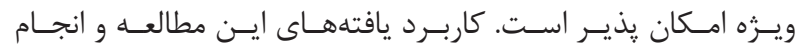

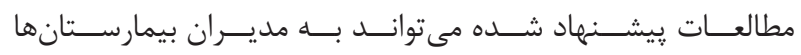

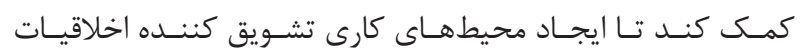

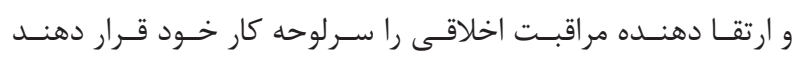

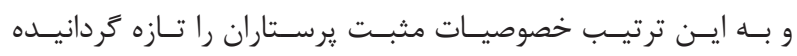

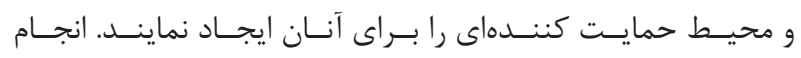

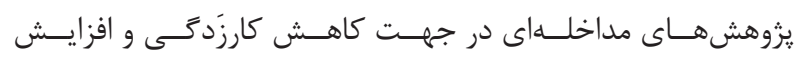

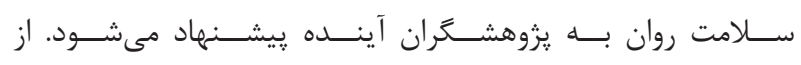

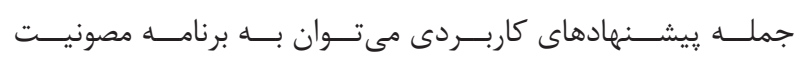

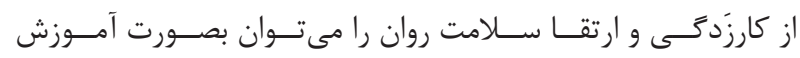

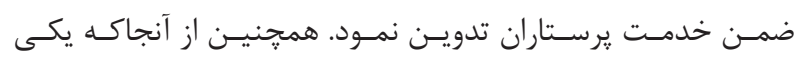

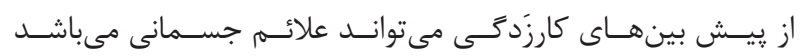

[Investigation of factors influencing burnout in staff colleges of the former University of Medical Sciences Iran]. Razi J Med Sci. 2010;18(90):2735.

12. Spoonerlane R. The Influence of work stress and work support on burnout inpublic hospital nurses. Queensland: Queensland Uniersity of Technology; 2004.

13. Hansen N, Sverke M, Naswall K. Predicting nurse burnout from demands and resources in three acute care hospitals under different forms of ownership: a cross-sectional questionnaire survey. Int J Nurs Stud. 2009;46(1):95-106. DOI: 10.1016/j.ijnurstu.2008.08.002 PMID: 18823625

14. Sadoc BJ, Sadock VA. Kaplan and Sadocks comprehensive text book of psychiatry. Baltimore: Lippincott Williams \& Wilkins; 2005.

15. Naderi N, Farnood A, Habibi M, Zojaji H, Balaii H, Firouzi F, et al. NOD2 exonic variations in Iranian Crohn's disease patients. Int J Colorectal Dis. 2011;26(6):775-81. DOI: $10.1007 /$ s00384-011-1145-4 PMID: 21274544

16. Jenkins R, Elliott P. Stressors, burnout and social support: nurses in acute mental health settings. J Adv Nurs. 2004;48(6):622-31. DOI: 10.1111/j.1365-2648.2004.03240.x PMID: 15548253

17. Godin I, Kittel F. Differential economic stability and psychosocial stress at work: associations with psychosomatic complaints and absenteeism. Soc Sci Med. 2004;58(8):1543-53. DOI: 10.1016/ S0277-9536(03)00345-9 PMID: 14759697

18. Ghaddar A, Mateo I, Sanchez P. Occupational stress and mental health among correctional officers: a cross-sectional study. J Occup Health. 2008;50(1):92-8. PMID: 18285652

19. Aziznejad P, Seyed javad H. [Burnout and its causes in clinical nurses working at hospitals of Babol University of Medical Sciences]. Jj Babol Univ Med Sci. 2006;8(2):63-9.

20. Setoodeh asl N, Ekhtiari H. [Review and related factors in nurses and midwives at Semnan University of Medical Sciences]. J Kordestan Univ Med Sci. 2011;39(1):77-83.

21. Abdi FH, Kaviani H, Khaghanizadeh M. [Medical colledge magazine, 
medical science university of Tehran]. 2007;65(6):65-75

22. Karahmadi M, Esmaeili Dehaghi N. [Aggression and some of its demographic correlates in nurses of pediatric wards in hospitals affiliated to isfahan medical university].J Res Behav Sci. 2007;5(2):337.

23. Golparvar M, Vaseghi Z, Masahebi M, Javadian Z. [Interpersonal Conflict functions and Conflict Management in the link between stress and burnout with aggression]. J Contemroary Psychol. 2012;7(2):61-72.

24. McManus IC, Keeling A, Paice E. Stress, burnout and doctors' attitudes to work are determined by personality and learning style: a twelve year longitudinal study of UK medical graduates. BMC Med. 2004;2:29. DOI: 10.1186/1741-7015-2-29 PMID: 15317650

25. Parslow RA, Jorm AF, Christensen H, Broom DH, Strazdins L, RM DS. The impact of employee level and work stress on mental health and GP service use: an analysis of a sample of Australian government employees. BMC Public Health. 2004;4:41. DOI: 10.1186/14712458-4-41 PMID: 15456518

26. Zahedi far S, Najariyan B, Shekar kan H. [Develop and validate a scale to measure aggression]. J Educ Psychol. 2000;3(7):73-102.

27. Salahian AAH, Babamiri M, Asgari A. [Factors predictive of burnout syndrome]. Nurs Res. 2010;6(23):23-31.

28. Buss AH, Perry M. The aggression questionnaire. J Pers Soc Psychol. 1992;63(3):452-9. PMID: 1403624

29. Ozer EJ, Weinstein RS, Maslach C, Siegel D. Adolescent AIDS prevention in context: the impact of peer educator qualities and classroom environments on intervention efficacy. Am J Community Psychol. 1997;25(3):289-323. PMID: 9332965

30. Fornell C, Larcker DF. Structural equation models with unobservable variables and measurement error: Algebra and statistics. J Market Res. 1981;18(2):382-8. DOI: $10.2307 / 3151312$

31. Mahdiyanfar F, Kimiaei SA, Ghanbari Hashem Abadi BA. [Examining the Effectiveness of Psycho- Social Empowerment Based on Choice Theory on Reducing Depression andAggression in Women with Husbands in Prison in 2014]. Iran J Forens Med. 2014;21(3):167-77.

32. Queirós C, Kaiseler M, da Silva AL. Burnout as predictor of aggressivity among police officers. J Police Stud Cahiers Politiestud. 2013;1(2). 


\title{
Burnout as Psychological Syndrome of Predictors of Aggression at the Hospitals
}

\author{
Alireza Tashakorian Jahromi ${ }^{1}$, Seyed Aligholi Rowshan ${ }^{2, *}$, \\ Baqer Kord ${ }^{2}$
}

${ }^{1}$ PhD Student of Management, Department of Public Administration, Faculty of Management and Economic, University of Sistan and Baluchestan, Zahedan, Iran

${ }^{2}$ Associate Professor, Faculty of Management and Economic, University of Sistan and Baluchestan, Zahedan, Iran

* Corresponding author: Seyed Aligholi Rowshan, Associate Professor, Department of Public Administration, Faculty of Management and Economic, University of Sistan

DOI: $10.21859 / \mathrm{nmj}-25023$ and Baluchestan, Zahedan, Iran.E-mail: salirowshan@gmail.com

Received: 23.07.2016

Accepted: 26.08.2016

\begin{tabular}{l} 
Keywords: \\
Burnout \\
Aggression \\
Anger \\
Depersonalization \\
Emotional Exhaustion \\
\hline How to Cite this Article: \\
Tashakorian Jahromi A, Rowshan \\
S A, Kord B. Burnout as Psycho- \\
logical Syndrome of Predictors \\
of Aggression at the Hospitals. \\
Sci J Hamadan Nurs Midwifery \\
Fac. 2016;25(2):16-26.DOI: \\
10.21859/nmj-25023 \\
\hline
\end{tabular}

(C) 2017 Scientific Journal of Hamadan Nursing \& Midwifery Faculty

\begin{abstract}
Introduction: There is limited research on the relationship between burnout and aggression (as a reaction to burnout and not personalities). In this study, this relationship was investigated with the presented model. This study focused on the relationship between aggression and burnout by using regression analysis to determine the predictors of aggression.

Methods: In this analytical study, using simple random sampling and with the help of Cochran, about 82 of the nurses of internal, surgery, emergency, psychiatric, ICU, and CCU section were selected at social security hospital in zahedan, as the minimum required sample. The study was conducted during January 2016, and a questionnaire was completed by all candidates. The study tool was a standard questionnaire in three parts, including demographic, Maslach burnout dimensions (1997), and of Buss and Perry (1992), and evaluated the reliability and validity, and distributed among the sample. To analyze the data, Pearson correlation test and Friedman test, using the SPSS software and Smart PLS software, were used for two-stage partial least squares method and structural equation model to analyze the assumptions, confirmatory factor analysis and path analysis.

Results: According to the path factors, the significance was determined at $\mathrm{P}$ value $=0.01$ for burnout and its dimensions, including anger ( $\mathrm{sig}=0.001$, Reg=-0.599), aggression ( $\operatorname{sig}=0.001$, Reg=0.433) and hostility (sig=0.001, Reg=0.364). Also, the burnout dimensions, including depersonalisation ( $\mathrm{sig}=0.001$, Reg=0.419), emotional exhaustion ( $\mathrm{sig}=0.01, \mathrm{Reg}=0.189)$ and feelling of ineffectiveness ( $\mathrm{sig}=0.01, \mathrm{Reg}=0.234$ ) had similar effects on aggression. The results showed that depersonalization has the greatest impact on aggression. On the other hand, according to Friedman test results among burnout dimensions, emotional exhaustion among nurses of this hospital was the most significant. Also, burnout had an indirect effect on anger.

Conclusions: In order to control the interaction between burnout and aggression and also their dimensions, the booster conditions of working and environmental mental health must be improved and restrictive conditions of burnout as independent and effective variables and aggression consequently prepared in the organization.
\end{abstract}

